\title{
Über die aus dem grünen Chromchlorid(bromid)hydrat durch Silbersalze fällbaren Chlormengen.
}

Von

\author{
R. F. WeInLand und A. Koch.
}

Während nach PELIGOT ${ }^{1}$ und JöRGENSEN ${ }^{2}$ durch Silbernitrat aus Lösungen des grünen Chromchloridhydrats, $\mathrm{CrCl}_{3} .6 \mathrm{H}_{2} \mathrm{O}, 2 / 3$ des vorhandenen Chlors gefällt werden, beobachteten WERNER und GuBSER $^{3}$ bei genauerer Untersuchung des Chlorids (1901), dafs es von der Temperatur der Fällungsflüssigkeit abhängt, wie viel Chlor gefällt wird, und sie konstatierten, dafs bei $0^{0}$ die $1 / \mathrm{s}$ des vorhandenen Chlors entsprechende Menge niedergeschlagen wird. Dem gegenüber fand WyrodborF ${ }^{4}$ (1902), dafs $\%$ des vorhandenen Chlors gefällt werden, und er vermutet, da Werner und Gubser nur $1 / 3$ erhielten, dafs es mehrere Modifikationen des grünen Cbromchloridhydrats gebe.

Wir haben das Verhalten dieses Chlorids gegen Silbersalze ebenfalls untersucht und gefunden, dafs die Menge des gefällten Chlors weniger von der Temperatur, als vielmehr von der Natur und Menge des zur Fällung benutzten Silbersalzes und besonders von der Art und Menge der zugesetzten Säure abhängig ist.

Werner und GUBSER haben bei den Fällungen Salpetersäure zugesetzt (auf $0.4 \mathrm{~g}$ Chlorid etwa 15 Tropfen konzentrierte Säure) und daher rührt es nach unseren Versuchen, dafs nur $1 / 3$ des Chlors niedergeschlagen wird, ohne Zusatz von Salpetersäure werden auch

: Ann. Chim. Phys. [3] 14 (1845), 241.

2 Journ. prakt. Chem. [2] 25 (1882), 415.

${ }^{3}$ Ber. deutsch. chem. Ges. 34 (1901), 1579.

4 Bull. Soe. Ohim. 27 (1902), 672 Anmerkung. 
bei $0^{\circ}$ durch die von Werner und GUBSER angewendete Silbernitratmenge etwa $\%$ desselben ausgefällt. Diese Bedeutung des Salpetersäurzusatzes wird von WERNER und GUBSER nicht hervorgehoben, sie erwähnen ihn nur als einen solchen, wie er bei Chlorfällungen üblich ist.

Jenes Drittel allein wird nur gefällt bei Gegenwart von gewissen Säuren in bestimmter Menge. Von den von uns untersuchten Silbersalzen fällen alle ohne Zusatz von Säuren wenigstens $2 / 3$ des Chlors, die meisten mehr, einige das gesamte Chlor.

Nach WeRNER und Gobser ist die gefundene Chlormenge aufser von der Temperatur noch davon abhängig, wie rasch man das gefällte Chlorsilber abfiltriert und nur bei möglichster Beschleunigung dieser Operation soll man die 1 Chloratom entsprechende Menge finden, sonst werde mehr erhalten. Dieser Einflufs der Zeit besteht in der Tat, aber erst, wenn man vor der Filtration 10 Minuten wartet, zeigt er sich einigermafsen und er tritt hinter den des Säurezusatzes weit zurück (s. u. 300 und 312).

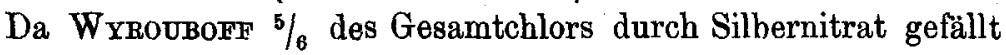
erhielt, vermuten wir, dals er keine Salpetersäure zusetzte (er sagt hierüber nichts); so werden bei einer bestimmten Silbernitratmenge $5 / 6$ niedergeschlagen (s. übrigens unten S. 301). Ein Grund zur Annahme mehrerer Modifikationen des grünen Chromchloridhydrats liegt daher hierin nicht. Einen Erklärungsversuch für den Befund von Peligot siehe weiter unten S. 309.

Wir haben dann ferner Fällungen in alkoholischer Lösung mit Silbernitrat ausgeführt und hierbei gefunden, dafs im Gegensatz $\mathrm{zu}$ den Fällungen in wässeriger Lösung ohne Zusatz von Säuren bei $0^{\circ}$ durch Silbernitrat in einigem Uberschufs das gesamte Chlor gefällt wird; fügt man Salpetersäure hinzu, so nimmt die Menge des sogleich niedergeschlagenen Chlors ab, aber sie bleibt stets über $2 / 3$ des Gesamtchlors.

Bei dem grünen Cäsium-Doppelsalz, $\mathrm{CrCl}_{3} \cdot 2 \mathrm{CsCl} .4 \mathrm{H}_{2} \mathrm{O}$ fanden Werner und GUBSER, dafs 3/5 des vorhandenen Chlors durch Silbernitrat gefällt werden; dies ist wiederum nur der Fall, wenn Salpetersäure nicht unter einer bestimmten Menge hinzugefügt wird, sonst fällt mehr heraus.

Bei dem grünen Chrombromidhydrat, welches dem Chlorid völlig entspricht, beobachteten WERNER und GUBSER, ${ }^{x}$ dafs unter

1 Ann. Chem. 322 (1902), 337. 
den beim Chlorid eingehaltenen Bedingungen $\left\langle 0^{\circ}\right.$, Zusatz von Salpetersäure) ${ }^{14} /{ }_{15}$ des vorhandenen Broms gefällt werden. Wir fanden bei diesem Salze, dals die verschiedenen Silbersalze allein sogleich das gesamte Brom fällen, dafs man aber durch Zusatz von gewissen Säuren die llenge des gefällten Broms bis auf $2 / 3$ des Gesamtbroms herunterdrücken kann.

\section{Versuchsanordnung.}

Es wurde mit Goochtiegeln gearbeitet, welche mit einer möglichst dünnen Asbestschicht beschickt waren; so konnte das Chlorsilber von der Fällungsflüssigkeit im Laufe von etwa 1 Min. abgesaugt werden. Die Fällungen machten wir in Erlenmeyerkölbchen, das Chromchlorid wurde kurz vor der Fällung in möglichst wenig Wasser gelöst und dann die Lösung des Silbersalzes, event. mit der betreffenden Säure versetzt, hinzugegeben und sofort filtriert und das Chlorsilber mit Wasser ausgewaschen. Das Filtrat wurde, um das nicht gefällte Chlor zu bestimmen, zuerst mit Ammoniak und dann mit Salpetersäure übersättigt, event. noch etwas Silbernitrat hinzugefügt und das nach mehrstündigem Erwärmen abgeschiedene Chlorsilber auf denselben Goochtiegel gebracht, durch welchen die erste Fällung abfiltriert worden war. Die Mehrzahl der Fällungen wurden bei $0^{\circ}$ ausgefürt, indem die Kölbchen mindestens $1 / 2$ Stunde in schmelzendes Eis gestellt wurden.

\section{Grünes Chromchloridhydrat, $\mathrm{CrCl}_{3} \cdot 6 \mathrm{H}_{2} \mathrm{O}$.}

Das zu den Versuchen benutzte Chlorid stellten wir nach dem Verfahren von RECOURA ${ }^{1}$ aus reiner Chromsäure her.

I. $0.2940 \mathrm{~g}$ Substanz: $0.4736 \mathrm{~g} \mathrm{AgCl}$ - $0.2980 \mathrm{~g}$ Substanz $0.0856 \mathrm{~g} \mathrm{Cr}_{2} \mathrm{O}_{\mathrm{s}}$.

II. $0.2404 \mathrm{~g}$ Substanz: $03882 \mathrm{~g} \mathrm{AgCl}$ - $-0.2388 \mathrm{~g}$ Substanz $0.0690 \mathrm{~g} \mathrm{Cr}_{2} \mathrm{O}_{3}$.

III. $0.4256 \mathrm{~g}$ Substanz: $0.6868 \mathrm{~g} \mathrm{AgCl}$. $-0.4050 \mathrm{~g}$ Substanz $0.1164 \mathrm{~g} \mathrm{Cr}_{2} \mathrm{O}_{\mathrm{s}}$.

\begin{tabular}{cccc} 
Berechnet für & \multicolumn{3}{c}{ Gefunden: } \\
$\mathrm{CrCl}_{8} .6 \mathrm{H}_{8} \mathrm{O}:$ & I & II & III \\
$\mathrm{Cr}=19.54$ & 19.67 & 19.78 & $19.65 \%$ \\
$\mathrm{Cl}=39.90$ & 39.83 & $\mathbf{3 9 . 9 2}$ & $\mathbf{3 9 . 9 0}$
\end{tabular}

Es wird von Peligot, Werner und Gubser als aus körnigen,

1 Ann. Chim. Phys. [6] 10 (1887), 21; siehe auch Werner und Gubser 1 c. S. 1594 , 
von Moberg ${ }^{1}$ als aus nadelförmigen, von Recoura als aus kleinen Kristallen bestehend beschrieben. Die von uns bei wiederholten Darstellungen erhaltenen Salze bildeten unter dem Mikroskop grüne, sehr gut ausgebildete, ungleich sechsseitige Täfelchen, welche nach freundlicher Mitteilung von Herrn Privatdozent Dr. Pliteninger optisch zweiachsig und von mono- oder asymmetrischem Typus sind.

Die wässerige Lösung aller von uns dargestellten, durch Waschen mit Aceton sowie Trocknen über Schwefelsäure und Natronkalk von anhängender Salzsäure sorgfältig befreiten Salzproben reagierte sauer, das Salz erleidet also hydrolytische Spaltung. Die alkoholische Lösung reagiert neutral.

\section{A. Fällungen mit verschiedenen Silbersalzen ohne Zusatz von Säuren.}

1. Mit Silbernitrat.

Tabelle 1 a.

Chlorfällungen im grünen Chromchloridhydrat durch steigende Mengen Sillbernitrat obne Zusatz von Säure bei $0^{\circ}$ und $50 \mathrm{ccm}$ Gesamtflüssigkeit; erste Fällung sogleich abfiltriert.

\begin{tabular}{|c|c|c|c|c|c|c|c|}
\hline \multirow{2}{*}{$\begin{array}{c}\mathrm{CrCl}_{\mathrm{s}} \\
6 \mathrm{H}_{2} \mathrm{O} \\
\mathrm{g}\end{array}$} & \multirow{2}{*}{$\begin{array}{l}\stackrel{\mathrm{a}}{\mathrm{AgNO}} \mathrm{N}_{3} \\
\text { berechnet } \\
\text { für At.Cl }\end{array}$} & \multicolumn{2}{|c|}{ Sofort gefällt } & \multicolumn{2}{|c|}{$\begin{array}{c}\text { b } \\
\text { Aus dem Filtrat } \\
\text { abgeschieden }\end{array}$} & \multirow{2}{*}{$\begin{array}{c}\text { Gesamt } \\
\text { Cl in } \\
\%\end{array}$} & \multirow[t]{2}{*}{$\mathrm{a} b$} \\
\hline & & $\mathrm{AgCl} g$ & $\mathrm{Cl} \%$ & $\mathrm{AgCl} g$ & Cl $\%$ & & \\
\hline 0.2134 & 3 & 0.2724 & 31.56 & 0.0724 & 8.39 & 39.95 & 25 \\
\hline 0.1950 & 4 & 0.2672 & 33.93 & 0.0484 & 6.13 & 40.06 & 24 \\
\hline 0.1966 & 6 & 0.2890 & 36.45 & 0.0298 & 3.74 & 40.19 & 22 \\
\hline 0.2072 & 8 & 0.3140 & 37.47 & 0.0214 & 2.55 & 40.02 & 19 \\
\hline 0.2176 & 9 & 0.3270 & 37.16 & 0.0240 & 2.72 & 39.98 & 24 \\
\hline 0.1942 & 12 & 0.2990 & 38.17 & 0.0138 & 1.75 & 39.92 & 21 \\
\hline 0.2006 & 18 & 0.3134 & 38.63 & 0.0106 & 1.30 & 39.93 & 23 \\
\hline 0.1952 & 24 & 0.3046 & 38.58 & 0.0104 & 1.32 & 39.90 & 31.6 \\
\hline 0.2076 & 45 & 0.3232 & 38.49 & 0.0108 & 1.24 & 39.73 & \\
\hline 0.2084 & 48 & 0.3220 & 38.21 & 0.0132 & 1.56 & 39.77 & \\
\hline $0.2044^{8}$ & 48 & 0.3212 & 38.86 & 0.0088 & 1.06 & 39.92 & \\
\hline
\end{tabular}

Berechnet für $1 \mathrm{Cl} 13.30 \%$, für $2 \mathrm{Cl} 26.60 \%$, für $3 \mathrm{Cl} 39.90 \%$.

Aus der Tabelle ist ersichtlich, dafs mit steigender Menge des Silbernitrats auch diejenige des sogleich gefällten Chlors zunimmt. Für 1 Atom Chlor berechnen sich $13.3 \%$, für 2 Atome $26.6 \%$ und

1 Dissert. de oxido chromoso. Helsingforss 1847; Gmelin-Kraut II, 2, 317.

2 Bei diesem Versuch betrug das Gesamtvolumen $100 \mathrm{ccm}$. 
für das gesamte Chlor 39.9\% $\%$ Von 3-18 Mol. Silbernitrat zeigt sich die interessante Beziehung, dafs die Mengen des zugesetzten Silbernitrats sich annähernd umgekehrt verhalten, wie die aus dem Filtrat der ersten Fällungen abgeschiedenen Chlormengen: Das Produkt $a b$ ist annähernd konstant (s. oben in der Tabelle). Von $24 \mathrm{Mol}$. Silbernitrat an trifft dies nicht mehr zu, es fällt weniger heraus als sich hiernach berechnen würde. Eigentümlich ist, dafs bei noch grölseren Mengen wieder weniger Chlor niedergeschlagen zu werden scheint; wir vermuteten zunächst, dies könnte von der Löslichkeit des Chlorsilbers in Silbernitratlösungen jener Konzentration herrühren; wir überzeugten uns aber durch blinde Versuche, dafs dies nicht der Fall ist.

Ein Versuch bei gewöhnlicher Temperatur mit 3 Mol. Silbernitrat ergab folgendes Resultat:

$0.2128 \mathrm{~g}$ Chlorid lieferten sogleich $0.2798 \mathrm{~g} \mathrm{AgCl}=32.51 \% \mathrm{Cl}$; aus dem Filtrat wurden erhalten $0.0652 \mathrm{~g} \mathrm{AgCl}=\frac{7.57 \%}{40.08 \%}$

Die Werte sind von den bei $0^{\circ}$ erhaltenen nicht sehr verschieden.

Die Verdünnung scheint hier von einem wenn auch kleinen Einfluis zu sein: Der letzte Wert der Tabelle wurde bei einer Gesamtflüssigkeitsmenge von $100 \mathrm{ccm}$ erhalten, es fiel dabei etwas mehr Chlor heraus.

Die folgende Tabelle zeigt den Einfluf's der Zeit auf die gefundenen Clorsilbermengen; filtriert man erst 20-30 Min. nach der

\section{Tabelle $1 \mathrm{~b}$.}

Chlorfällungen im grünen Chromchloridhydrat mit für 6 Atome Chlor berechnetem Silbernitrat ohne Zusatz von Säure. Nach Zusatz des Silbernitrats wurde nicht sogleich, sondern erst nach einiger Zeit abfiltriert. Temperatur $0^{\circ}$.

Gesamtvolumen $50 \mathrm{cem}$.

\begin{tabular}{|c|c|c|c|c|c|c|c|}
\hline \multirow{2}{*}{$\begin{array}{c}\mathrm{CrCl}_{3} \cdot 6 \mathrm{H}_{2} \mathrm{O} \\
\mathrm{g}\end{array}$} & \multirow{2}{*}{\multicolumn{2}{|c|}{$\begin{array}{c}\text { Zeit des } \\
\text { Stehenlassens } \\
\text { vor dem } \\
\text { Abfiltrieren }\end{array}$}} & \multicolumn{2}{|c|}{ Sofort gefällt } & \multicolumn{2}{|c|}{$\begin{array}{c}\text { Aus dem Filtrat } \\
\text { abgeschieden }\end{array}$} & \multirow{2}{*}{$\begin{array}{l}\text { Gesamt } \\
\text { Chlor } \\
\text { in } \%\end{array}$} \\
\hline & & & $\mathrm{AgCl} \mathrm{g}$ & $\mathrm{Cl} \%$ & $\mathrm{AgCl} g$ & $\mathrm{Cl} \%$ & \\
\hline 0.2012 & & Minut. & 0.2980 & 36.62 & 0.0264 & 3.24 & 39.86 \\
\hline 0.2054 & 5 &, & 0,3080 & 37.08 & 0.0248 & 2.98 & 40.06 \\
\hline 0.2094 & 15 & , & 0.3152 & 37.22 & 0.0230 & 2.71 & 39.93 \\
\hline 0.2122 & 20 & , & 0.3240 & 37.75 & 0.0186 & 2.16 & 39.91 \\
\hline 0.1990 & 30 & , & 0.3054 & 37.95 & 0.0160 & 1.98 & 39.93 \\
\hline
\end{tabular}


Fällung, so findet man etwa $1 \%$ Chlor mehr als bei sofortiger Filtration, bezw. man findet dann bei $6 \mathrm{Mol}$. Silbernitrat soviel Chlor, als man bei etwa $10 \mathrm{Mol}$. Silbernitrat bei sofortiger Filtration finden würde.

Unter diese Versuchsreihe rechnen wir, wie S. 297 erwähnt, den Befund von WrвопвоF, dafs durch Silbernitrat $5 / 6$ des Chlors gefällt werden. WYrouboFr fand sogleich niedergeschlagen $36.6 \%$, aus dem Filtrat erhielt er noch $7 \%$, zusammen $43.6 \%$ statt der berechneten 39.9. Den Überschufs erklärt W YrouboFr damit, dafs Chlorsilber mit dem Chromchlorid in der Kälte eine in Wasser schwer lösliche Verbindung gebe; wir haben nie dergleichen beobachtet. WxrourofF nimmt demgemäls den ersten Wert als um etwa $3.5 \%$ (!) zu hoch an und findet als erste Fällung $33 \%=5 / 6$ des Gesamtchlors. Diese Annahme ist ziemlich willkürlich, aufserdem fehlen Angaben über Temperatur (es heilst nur à froid) und über die Menge des Silbernitrats, so dals das Resultat nicht mit einem der Werte der obigen Tabelle vergleichbar ist.

2. Mit Silbersulfat.

Tabelle 2.

Chlorfällungen im grünen Chromehloridhydrat durch steigende Mengen Silbersulfat ohne Zusatz von Säure bei $0^{\circ}$ und $60 \mathrm{ccm}$ Gesamtvolumen; erste Fallung sofort abfiltriert.

\begin{tabular}{|c|c|c|c|c|c|c|}
\hline \multirow{2}{*}{$\begin{array}{c}\mathrm{CrCl}_{3} \cdot 6 \mathrm{H}_{2} \mathrm{O} \\
\mathrm{g}\end{array}$} & \multirow{2}{*}{$\begin{array}{l}\mathrm{Ag}_{2} \mathrm{SO}_{4} \text { be- } \\
\text { rechnet für } \\
\text { Atome Chlor }\end{array}$} & \multicolumn{2}{|c|}{ Sofort gefälltt } & \multicolumn{2}{|c|}{$\begin{array}{c}\text { Aus dem Filtrat } \\
\text { abgeschieden }\end{array}$} & \multirow{2}{*}{$\begin{array}{c}\text { Gesamt- } \\
\text { chlor } \\
\text { in } \%\end{array}$} \\
\hline & & $\mathrm{AgCl} g$ & Cl $\%$ & AgCl $\mathrm{g}$ & $\mathrm{Cl} \%$ & \\
\hline 0.1052 & 3 & 0.1572 & 36.95 & 0.0132 & 3.10 & 40.05 \\
\hline 0.1006 & 4 & 0.1604 & 39.42 & 0.0024 & 0.58 & 40.00 \\
\hline 0.1188 & 6 & 0.1896 & 39.46 & 0.0028 & 0.58 & 40.04 \\
\hline 0.1050 & 9 & 0.1690 & 39.80 & 0 & 0 & 39.80 \\
\hline 0.1062 & 9 & 0.1718 & 40.00 & 0 & 0 & 40.00 \\
\hline
\end{tabular}

Während bei den Fällungen mit Silbernitrat auch bei ziemlich grofsem Überschufs von diesem bei der ersten Fällung nicht sämtliches Chlor gefällt wurde, ist dies beim Silbersulfat schon der Fall, wenn das Dreifache des Berechneten zugesetzt wird; aber bereits bei Fällung mit einem Überschufs von nur $1 / 3$ wird beinahe das gesamte Chlor niedergeschlagen, auch die für 3 Atome Chlor berechnete Menge Silbersulfat fällt wesentlich mehr als die entsprechende Menge Silbernitrat. Es zeigt sich hier und bei den fol- 
genden Salzen deutlich der Einflufs des Anions des Silbersalzes auf die Menge des gefällten Chlorsilbers.

3. Mit Silberchlorat.

Tabelle 3.

Chlorfällungen im grünen Chromchloridhydrat durch steigende Mengen Silberchlorat ohne Zusatz von Saure; Temperatur $0^{\circ}$, Gesamtvolumen $50 \mathrm{ccm}$. Erste Fällung sofort abfiltriert.

\begin{tabular}{|c|c|c|c|c|c|c|}
\hline \multirow{2}{*}{$\begin{array}{c}\mathrm{CrCl}_{3} .6 \mathrm{H}_{2} \mathrm{O} \\
\mathrm{g}\end{array}$} & \multirow{2}{*}{$\begin{array}{l}\mathrm{AgClO}_{3} \text { be- } \\
\text { rechnet für } \\
\text { Atome Chlor }\end{array}$} & \multicolumn{2}{|c|}{ Sofort gefällt } & \multicolumn{2}{|c|}{$\begin{array}{c}\text { Aus dem Filtrat } \\
\text { abgeschieden }\end{array}$} & \multirow{2}{*}{$\begin{array}{l}\text { Gesant } \\
\text { chlor } \\
\text { in } \%\end{array}$} \\
\hline & & $\mathrm{AgCl} \mathrm{g}$ & $\mathrm{Cl} \%$ & $\mathrm{AgCl} g$ & $\mathrm{Cl} \%$ & \\
\hline 0.2008 & 3 & 0.2514 & 30.95 & 0.0728 & 8.96 & 39.91 \\
\hline 0.2094 & 4 & 0.2704 & 31.93 & 0.0684 & 8.07 & 40.00 \\
\hline 0.2096 & 6 & 0.2834 & 33.43 & 0.0564 & 6.65 & 40.08 \\
\hline 0.2068 & 12 & 0.2960 & 30.39 & 0.0406 & 4.85 & $40.24^{2}$ \\
\hline $0.2112^{1}$ & 24 & 0.3108 & 36.39 & 0.0348 & 4.07 & $40.46^{2}$ \\
\hline $0.2200^{1}$ & 48 & 0.3164 & 35.56 & 0.0496 & 5.57 & $41.13^{2}$ \\
\hline
\end{tabular}

Das Silberchlorat verhält sich ähnlich wie das Nitrat (S. 299). Die Menge des sogleich gefällten Chlors steigt mit zunehmendem Silberchlorat und bei einer gewissen Menge (48 Mol.) wird wieder weniger gefällt. Dabei sind die gefällten Chlormengen kleiner als beim Nitrat. Auch die Verdünnung hat hier einigen Einflufs.

4. Mit Silberperchlorat.

Tabelle 4 a.

Chlorfällungen im grünen Chromchloridhydrat durch steigende Mengen Silberperchlorat ohne Zusatz von Säure bei $0^{\circ}$ und $25 \mathrm{ccm}$ Gesamtvolumea; die erste Fillung wurde sofort abfiltriert.

\begin{tabular}{|c|c|c|c|c|c|c|}
\hline \multirow{2}{*}{$\begin{array}{c}\mathrm{CrCl}_{3} \cdot 6 \mathrm{H}_{2} \mathrm{O} \\
\mathrm{g}\end{array}$} & \multirow{2}{*}{$\begin{array}{l}\mathrm{AgClO}_{4} \text { be- } \\
\text { rechnet für } \\
\text { Atome Chlor }\end{array}$} & \multicolumn{2}{|c|}{ Sofort gefällt } & \multicolumn{2}{|c|}{$\begin{array}{c}\text { Aus dem Filtrat } \\
\text { abgeschieden }\end{array}$} & \multirow{2}{*}{$\begin{array}{c}\text { Gesamt- } \\
\text { chlor } \\
\text { in } \%\end{array}$} \\
\hline & & $\mathrm{AgCl} \mathrm{g}$ & $\mathrm{Cl} \%$ & $\mathrm{AgCl} \mathrm{g}$ & Cl $\%$ & \\
\hline 0.0988 & 3 & 0.1148 & 28.73 & 0.0446 & 11.16 & 39.89 \\
\hline 0.1088 & 6 & 0.1210 & 27.50 & 0.0548 & 12.45 & 39.95 \\
\hline 0.1080 & 9 & 0.1190 & 27.35 & 0.0542 & 12.41 & 39.66 \\
\hline 0.1040 & 12 & 0.1110 & 26.39 & 0.0562 & 13.36 & 39.75 \\
\hline 0.1027 & 24 & 0.1088 & 26.20 & 0.0567 & 13.65 & 39.85 \\
\hline 0.1026 & 48 & 0.1082 & 26.08 & 0.0573 & 13.81 & 39.89 \\
\hline
\end{tabular}

${ }^{1}$ Bei diesen Versuchen betrug das Gesamtyolumen $100 \mathrm{ccm}$.

- Das Gesamtchlor ist bei den drei letzten Bestimmungen deshalb zu hoch, weil beim Erwärmen des Filtrats mit Salpetersäure das überschüssige Silberchlorat teilweise zersetzt wird. 
Tabelle $4 \mathrm{~b}$.

Chlorfällungen im grünen Chromchloridhydrat durch steigende Mengen Silberperchlorat ohne Zusatz von Säure; Gesamtvolumen $100 \mathrm{ccm}$, Temperatur $0^{\circ}$.

Die erste Fällung warde sofort abfiltriert.

\begin{tabular}{|c|c|c|c|c|c|c|}
\hline \multirow{2}{*}{$\begin{array}{c}\mathrm{CrCl}_{3} \cdot 6 \mathrm{H}_{2} \mathrm{O} \\
\mathrm{g}\end{array}$} & \multirow{2}{*}{$\begin{array}{l}\mathrm{AgClO}_{4} \text { be- } \\
\text { rechnet für } \\
\text { Atome Chlor }\end{array}$} & \multicolumn{2}{|c|}{ Sofort gefällt } & \multicolumn{2}{|c|}{$\begin{array}{l}\text { Aus dem Filtrat } \\
\text { abgeschieden }\end{array}$} & \multirow{2}{*}{$\begin{array}{l}\text { Gesamt- } \\
\text { ehlor } \\
\text { in } \%\end{array}$} \\
\hline & & $\mathrm{AgCl} \mathrm{g}$ & Cl $\%$ & AgCI $g$ & Ci $\%$ & \\
\hline 0.1068 & 3 & 0.1390 & 32.18 & 0.0324 & 7.50 & 39.68 \\
\hline 0.1080 & 6 & 0.1306 & 29.90 & 0.0428 & 9.80 & 39.70 \\
\hline 0.1050 & 9 & 0.1172 & 27.60 & 0.0518 & 12.20 & 39.80 \\
\hline 0.1046 & 12 & 0.1160 & 27.42 & 0.0520 & 12.29 & 39.71 \\
\hline 0.1028 & 24 & 0.1140 & 27.42 & 0.0520 & 12.51 & 39.93 \\
\hline 0.1016 & 48 & 0.1180 & 28.72 & 0.0456 & 11.10 & 39.82 \\
\hline
\end{tabular}

Hier zeigt sich die eigentümliche Tatsache, dafs durch Steigerung des Silberperchlorats die Menge des sogleich gefällten Chlorsilbers sich etwas vermindert. Bei einem Gesamtvolumen von $25 \mathrm{ccm}$ auf $0.1 \mathrm{~g}$ Chromchlorid werden von 6 bis $48 \mathrm{Mol}$. Silberperchlorat annähernd $2 / 3$ des vorhandenen Chlors gefällt (berechnet $26.6 \%$ ). Bei einer Verdünnung von $100 \mathrm{ccm}$ (Tabelle $4 \mathrm{~b}$ ) wird diese Menge von 9 bis $24 \mathrm{Mol}$. Silberperchlorat niedergeschlagen. Aber auch 48 Mol. fällen kaum nennenswert mehr. Durch entsprechende Mengen Silbernitrat wurde viel mehr gefällt.

Blinde Versuche zeigten, dafs Chlorsilber in Lösungen von Silberperchlorat der angegebenen Konzentration unlöslich ist. Die Verminderung des gefällten Chlorsilbers mit steigender Silberperchloratmenge kann somit nicht hierher rühren.

5. Mit Silberpermanganat.

Tabelle 5 .

Chlorfällungen im grünen Chromehloridhydrat durch steigende Mengen Silberpermanganat ohne Zusatz von Säure bei $0^{\circ}$ und $80 \mathrm{ccm}$ Gesamtvolumen. Die erste Fällung wurde sogleich abfiltriert.

\begin{tabular}{|c|c|c|c|c|c|c|}
\hline \multirow{2}{*}{$\begin{array}{c}\mathrm{CrCl}_{3} \cdot 6 \mathrm{H}_{2} \mathrm{O} \\
\mathrm{g}\end{array}$} & \multirow{2}{*}{$\begin{array}{l}\mathrm{AgMnO} \\
\text { rechnet für } \\
\text { Atome Chlor }\end{array}$} & \multicolumn{2}{|c|}{ Sofort gefällt } & \multicolumn{2}{|c|}{$\begin{array}{c}\text { Im Filtrat } \\
\text { abgeschieden }\end{array}$} & \multirow{2}{*}{$\begin{array}{c}\text { Gesamt } \\
\text { chlor } \\
\text { in } \%\end{array}$} \\
\hline & & $\mathrm{AgCl} \mathrm{g}$ & $\mathrm{Cl} \%$ & $\mathrm{AgCl} \mathrm{g}$ & Cl $\%$ & \\
\hline 0.1334 & 3 & 0.1566 & 29.03 & 0.0600 & 11.12 & 40.15 \\
\hline 0.0886 & 6 & 0.1264 & 35.27 & 0.0170 & 4.74 & 40.01 \\
\hline
\end{tabular}


Silberpermanganat verhält sich ähnlich dem Nitrat; mit steigender Menge desselben nimmt auch diejenige des gefällten Chlorsilbers $z u$, und die Werte des letzteren sind etwa so grofs wie beim Silbernitrat.

Bei diesen Fällungen schied sich meistens etwas Mangansuperoxyd ab und mischte sich dem Chlorsilber bei. Wir reinigten dieses sogleich nach dem Abfiltrieren davon durch Behandeln mit schwefliger Säure.

6. Mit Silbernitrit.

Tabelle 6 a.

Chlorfällungen im grünen Chromchloridhydrat durch steigende Mengen Silbernitrit ohne Säure bei $0^{\circ} ; 50 \mathrm{ccm}$ Gesamtvolumen. Erste Fällung sofort abfiltriert.

\begin{tabular}{|c|c|c|c|c|c|c|}
\hline \multirow{2}{*}{$\begin{array}{c}\mathrm{CrCl}_{3} \cdot 6 \mathrm{H}_{2} \mathrm{O} \\
g\end{array}$} & \multirow{2}{*}{$\begin{array}{l}\mathrm{AgNO}_{2} \text { be- } \\
\text { rechnet für } \\
\text { Atome Chlor }\end{array}$} & \multicolumn{2}{|c|}{ Sofort gefällt } & \multicolumn{2}{|c|}{$\begin{array}{c}\text { Im Filtrat } \\
\text { abgeschieden }\end{array}$} & \multirow{2}{*}{$\begin{array}{l}\text { Gesamt- } \\
\text { chlor } \\
\text { in } \%\end{array}$} \\
\hline & & $\operatorname{AgCl} \mathrm{g}$ & Cl $\%$ & $\mathrm{AgCl} g$ & $\mathrm{Cl} \%$ & \\
\hline 0.0560 & 3 & 0.0792 & 34.97 & 0.0108 & 4.76 & 39.73 \\
\hline 0.0574 & 4 & 0.0876 & 37.73 & 0.0052 & 2.24 & 39.97 \\
\hline 0.0538 & 6 & 0.0844 & 38.79 & 0.0026 & 1.19 & 39.98 \\
\hline
\end{tabular}

Tabelle $6 \mathrm{~b}$.

Ebenso wie in Tabelle 6 a, aber bei Zimmertemperatur.

\begin{tabular}{|c|c|c|c|c|c|c|}
\hline \multirow{2}{*}{$\begin{array}{c}\mathrm{CrCl}_{3} \cdot 6 \mathrm{H}_{2} \mathrm{O} \\
\mathrm{g}\end{array}$} & \multirow{2}{*}{$\begin{array}{l}\mathrm{AgNO} \\
\text { rechnet für } \\
\text { Atome Chlor }\end{array}$} & \multicolumn{2}{|c|}{ Sofort gefällt } & \multicolumn{2}{|c|}{$\begin{array}{c}\text { Im Filtrat } \\
\text { abgeschieden }\end{array}$} & \multirow{2}{*}{$\begin{array}{c}\text { Gesamt- } \\
\text { chlor } \\
\text { in } \%\end{array}$} \\
\hline & & $\mathrm{AgCl} \mathrm{g}$ & $\mathrm{Cl} \%$ & $\mathrm{AgCl} \mathrm{g}$ & $\mathrm{Cl} \%$ & \\
\hline 0.0570 & 3 & 0.0866 & 37.57 & 0.0052 & 2.25 & 39.82 \\
\hline 0.0532 & 4 & 0.0858 & 39.88 & 0 & 0 & 39.88 \\
\hline 0.0510 & 6 & 0.0820 & $39.7 \tilde{~}$ & 0 & 0 & 39.75 \\
\hline
\end{tabular}

Mit Zusatz steigender Mengen von Silbernitrit nimmt die Menge des sogleich gefällten Chlors rasch zu, bei gewöhnlicher Temperatur wird schon durch $4 \mathrm{Mol}$. Nitrit das gesamte Chlor gefällt.

7. Mit Silberlaktat.

\section{Tabelle 7 .}

Chlorfällungen im grünen Chromchloridhydrat durch steigende Mengen Silberlak tat ohne Säure bei $0^{\circ}$; Gesamatvolumen 30 cem. Erste Fällung sofort abfiltriert.

\begin{tabular}{|c|c|c|c|c|c|c|}
\hline \multirow{2}{*}{$\begin{array}{c}\mathrm{CrCl}_{3} \cdot 6 \mathrm{H}_{2} \mathrm{O} \\
\mathrm{g}\end{array}$} & \multirow{2}{*}{$\begin{array}{l}\mathrm{AgC}_{3} \mathrm{H}_{5} \mathrm{O}_{3} \\
\text { berechnet f. } \\
\text { Atome Chlor }\end{array}$} & \multicolumn{2}{|c|}{ Sofort gefällt } & \multicolumn{2}{|c|}{$\begin{array}{c}\text { Im Filtrat } \\
\text { abgeschieden }\end{array}$} & \multirow{2}{*}{$\begin{array}{c}\text { Gesamt- } \\
\text { chlor } \\
\text { in } \%\end{array}$} \\
\hline & & $\mathrm{AgCl} g$ & Cl $\%$ & $\mathrm{AgCl} \mathrm{g}$ & $\mathrm{Cl} \%$ & \\
\hline 0.1070 & 3 & 0.1684 & 38.92 & 0.0034 & 0.79 & 39.71 \\
\hline 0.1022 & 4. & 0.1646 & 39.82 & 0 & 0 & 39.82 \\
\hline 0.1088 & 6 & 0.1756 & 39.91 & 0 & 0 & 39.91 \\
\hline
\end{tabular}


Durch 3 Mol. Silberlaktat wird beinahe das gesamte Chlor sogleich gefällt, bei $4 \mathrm{Mol}$. ist dies der Fall. Silberlaktat verhält sich dem Acetat (s. das folgende) sehr ähnlich.

8. Mit Silberacetat.

Tabelle 8.

Chlorfallungen im grïnen Chromchloridhydrat mit Silberacetat ohne Säure bei $0^{\circ}$ und Zimmertemperatur; Gesamtlüssigkeit $50 \mathrm{ccm}$; nach Zusatz des Silberacetats wird sofort abfiltriert.

\begin{tabular}{c|c|c|c|c|c}
\hline $\begin{array}{c}\mathrm{CrCl}_{8} \cdot 6 \mathrm{H}_{2} \mathrm{O} \\
\mathrm{g}\end{array}$ & $\begin{array}{c}\text { Temperatur } \\
\text { in }\end{array}$ & $\begin{array}{c}\text { Silberacetat ber. } \\
\text { für Atome } \mathrm{Cl}\end{array}$ & $\begin{array}{c}\text { Sofort gefälit } \\
\mathrm{AgCl} \mathrm{g}\end{array}$ & $\begin{array}{c}\text { Im Filtrat abge- } \\
\mathrm{Cl} \%\end{array}$ & $\begin{array}{c}\text { Amied. AgCl } \mathrm{g} \\
\text { schied }\end{array}$ \\
\hline
\end{tabular}

Das Silberacetat ist das einzige von uns beobachtete Silbersalz, welches bei $0^{\circ}$ (und bei gewöhnlicher Temperatur) in für 3 Atome Chlor berechneter Menge das gesamte Chlor des grünen Chromchloridbydrats sogleich fällt.

Von den untersuchten Silbersalzen fällt somit, wenn keine Säure zugesetzt wird, das Acetat in für 3 Atome Chlor berechneter Menge sogleich das gesamte Chlor des Chromchloridhydrats, das Lactat, das Nitrit, das Sulfat tun dies, wenn sie in einigem Überschufs zugesetzt werden. Dagegen fällen das Nitrat, das Chlorat und das Perchlorat auch in sehr grofsem Überschuls das gesamte Chlor nicht vollständig. Am wenigsten fällt das. Perchlorat, nämlich etwa $2 / 3$ des vorhandenen Chlors, die anderen fällen mehr. Eigentümlich ist die Erscheinung, dals beim Perchlorat durch Steigerung dieses die Menge des sogleich gefällten Chlorsilbers sich vermindert; bei den. anderen ist dies erst bei sehr stark vermehrtem Zusatz des Silbersalzes der Fall.

Im allgemeinen fällen hiernach die Silhersalze schwacher Säuren, wie Essigsäure, Milchsäure, salpetrige Säure, sowie das Silbersalz der ziemlich starken Schwefelsäure, in genügendem Überschul's das Chlor des grünen Chromchloridhydrats vollständig, während die Silbersalze der starken Säuren nur einen Teil des Chlors fällen.

\section{B. Fällungen bei Gegenwart der Säure des betr. Silbersalzes.}

Bei den im obigen beschriebenen Versuchen hatte sich gezeigt, dals die Menge des sogleich gefällten Chlors einerseits von der 
Natur des Silbersalzes, andererseits von dessen Menge abhängig ist. Des weiteren fanden wir, dafs sie ganz besonders auch von der Menge der zugesetzten Säure bestimmt wird. Es sind also zwei Momente, von denen es abhängt, wie viel Chlor man bei der ersten Fällung erhält, nämlich erstens die Menge des Silbersalzes, und zweitens diejenige der Säure. Die folgenden Versuche wurden daher so angestellt, dafs einmal bei gleichbleibender Anzahl der Moleküle des Silbersalzes die Säure gesteigert wurde, sodann bei gleichbleibender Säuremenge das Silbersalz vermehrt wurde.

9. Mit Silbernitrat unter Zusatz von Salpetersäure.

\section{Tabelle 9 a.}

Chlorfällungen im grünen Chromehloridhydrat mit für 3 Atome Chlor berechnetem Silbernitrat unter Zusatz steigender Mengen Salpetersäure.

Temperatur $0^{\circ}$, Gesamtvolumen $50 \mathrm{ccm}$. Erste Fällung sogleich filtriert.

\begin{tabular}{|c|c|c|c|c|c|c|}
\hline $\mathrm{CrCl}_{3} \cdot 6 \mathrm{H}_{2} \mathrm{O}$ & $\begin{array}{l}\mathrm{HNO}_{3} \text { Mol. } \\
\text { auf } 1 \text { Mol. }\end{array}$ & \multicolumn{2}{|c|}{ Sofort gefällt } & \multicolumn{2}{|c|}{$\begin{array}{c}\text { Im Filtrat } \\
\text { abgeschieden }\end{array}$} & $\begin{array}{l}\text { Gesamt } \\
\text { chlor }\end{array}$ \\
\hline & $\mathrm{CrCl}_{3} \cdot 6 \mathrm{H}_{2} \mathrm{O}$ & $A g C l g$ & $\mathrm{Cl} \%$ & $\mathrm{AgCl} \mathrm{g}$ & Cl $\%$ & in $\%$ \\
\hline 0.2134 & 0 & 0.2724 & 31.56 & 0.0724 & 8.39 & 39.95 \\
\hline 0.2104 & 0.04 & 0.2562 & 30.11 & 0.0844 & 9.91 & 40.02 \\
\hline 0.2100 & 0.08 & 0.2390 & 28.14 & 0.1004 & 11.82 & 39.96 \\
\hline 0.2038 & 0.13 & 0.2216 & 26.89 & 01074 & 13,03 & 39.92 \\
\hline 0.1990 & 0.17 & 0.1964 & 24.40 & 0.1242 & 15.48 & 39.83 \\
\hline 0.2130 & 0.24 & 0.2010 & 23.33 & 0.1424 & 16.53 & 39.86 \\
\hline 0.2124 & 0.3 & 0.1752 & 20.40 & 0.1674 & 19.49 & 39.89 \\
\hline 0.2106 & 0.4 & 0.1568 & 18.41 & 0.1842 & 21.62 & 40.03 \\
\hline 0.2076 & 0.8 & 0.1436 & 17.10 & 0.1910 & 22.75 & 39.85 \\
\hline 0.2158 & 1.6 & 0.1368 & 15.67 & 0.2126 & 24.36 & 40.03 \\
\hline 0.2056 & 2.5 & 0.1230 & 14.80 & 0.2094 & 25.18 & 39.98 \\
\hline 0.1964 & 3.5 & 0.1142 & 14.38 & 0.2032 & 25.58 & 39.96 \\
\hline 0.2004 & 4.3 & 0.1170 & 14.43 & 0.2072 & 25.56 & 39.99 \\
\hline 0.2112 & 5.7 & 0.1212 & 14.19 & 0.2200 & 25.75 & 39.94 \\
\hline 0.2060 & 10.4 & 0.1172 & 14.07 & 0.2162 & 25.95 & 40.02 \\
\hline 0.2070 & 15.7 & 0.1172 & 14.00 & 0.2172 & 25.94 & 39.94 \\
\hline 0.2006 & 24.4 & 0.1162 & 14.32 & 0.2074 & 25.57 & 39.89 \\
\hline 0.2174 & 37.5 & 0.1262 & 14.35 & 0.2244 & 25.52 & 39.87 \\
\hline 0.2005 & 57 & 0.1186 & 1463 & 0.2050 & 25.28 & 39.91 \\
\hline 0.2080 & 79 & 0.1230 & 14.62 & 0.2144 & 25.49 & 40.11 \\
\hline 0.2148 & 114 & 0.1302 & 14.99 & 0.2140 & 24.64 & 39.63 \\
\hline 0.2076 & 157 & 0.1292 & 15.39 & 0.2048 & 24.39 & 39.78 \\
\hline 0.2042 & 239 & 0.1296 & 15.69 & 0.2000 & 24.22 & 39.91 \\
\hline 0.1994 & 313 & 0.1406 & 17.44 & 0.1814 & 22.49 & 39.93 \\
\hline 0.2040 & 408 & 0.1490 & 18.06 & 0.1812 & 21.96 & 40.02 \\
\hline 0.2054 & 507 & 0.1723 & 20.74 & 0.1593 & 19.18 & 39.92 \\
\hline 0.2068 & 604 & 0.1912 & 22.86 & 0.1434 & 17.15 & 40.01 \\
\hline 0.2170 & 877 & 0.3214 & 36.62 & 0.0302 & 3.44 & 40.06 \\
\hline
\end{tabular}


Man sieht, wie grols der Einflufs der Säure ist: Schon etwa $1 / 8$ Mol. Salpetersäure auf $1 \mathrm{Mol}$. Chromchloridhydrat hat die Folge, dafs nur $2 / 3(26.6 \%)$ des vorhandenen Chlors gefällt werden, und bei fernerer Steigerung der Säure sinkt die Menge des gefällten Chlors rasch auf etwa $1 / 3(13.3 \%)$, welcher Wert bei etwa $6 \mathrm{Mol}$. Salpetersäure erreicht wird. Vermehrt man die Salpetersäure noch mehr, so geht zunächst das Chlor nicht weiter herunter, sondern man findet noch bei etwa $50 \mathrm{Mol}$. gleich viel, dann aber steigt das Chlor wieder langsam, indessen fallen selbst bei $600 \mathrm{Mol}$. erst etwa $3 / 5$ des gesamten Chlors heraus. Bei Zusatz ron noch mehr Salpetersäure wirkt diese auf die Salzsäure schwach zersetzend ein, der letzte Wert der Tabelle ist daher etwas unsicher.

Werner und Gubser ${ }^{1}$ fügten auf etwa $0.4 \mathrm{~g}$ Chromchloridhydrat 15 Tropfen konzentrierter Salpetersäure hinzu und fällten mit $1 \mathrm{~g}$ Silbernitrat; die konzentrierte Salpetersäure zu $68 \%$ gerechnet und 15 Tropfen als $1 \mathrm{~g}$, ergibt auf $1 \mathrm{Mol}$. Chromchlorid etwa $3.9 \mathrm{Mol}$. Silbernitrat und etwa $7 \mathrm{Mol}$. Salpetersäure. Sie finden bei möglichst raschem Filtrieren in 4 Versuchen $15.3-15.6 \%$ Chlor. Da aber die Berechnung der Salpetersäure etwas willkürlich ist, lassen sich diese Werte nicht direkt mit den unserigen vergleichen (wir erhielten bei $7 \mathrm{Mol}$. Salpetersäure rund $14.0 \%$ Chlor). Dagegen stimmen die von Werner und GubsER beim Titrieren unter denselben Bedingungen erhaltenen Werte mit den unserigen überein.

Die Werte der folgenden Tabelle erhielten wir unter denselben Bedingungen wie die der vorhergehenden, nur mit dem Unterschied, dafs bei gewöhnlicher Temperatur gearbeitet wurde.

\section{Tabelle $9 \mathrm{~b}$.}

Chlorfăllungen im grünen Chromchloridhydrat mit für 3 Atome Chlor berechnetem Silbernitrat unter Zusatz steigender Mengen Salpetersäure. Zimmertemperatur. Gesamtvolumen $50 \mathrm{ccm}$. Erste Fällung sofort abfiltriert.

\begin{tabular}{|c|c|c|c|c|c|c|}
\hline \multirow{2}{*}{$\begin{array}{c}\mathrm{CrCl}_{3} \cdot 6 \mathrm{H}_{2} \mathrm{O} \\
\mathrm{g}\end{array}$} & \multirow{2}{*}{$\begin{array}{l}\mathrm{HNO}_{3} \text { in Mol. } \\
\text { auf } 1 \mathrm{Mol} \text {. } \\
\mathrm{CrCl}_{3} .6 \mathrm{H}_{2} \mathrm{O}\end{array}$} & \multicolumn{2}{|c|}{ Sofort gefällt } & \multicolumn{2}{|c|}{$\begin{array}{c}\text { Im Filtrat } \\
\text { ausgeschieden }\end{array}$} & \multirow{2}{*}{$\begin{array}{c}\text { Gesamt- } \\
\text { ehlor } \\
\text { in } \%\end{array}$} \\
\hline & & $\mathrm{AgCl} \mathrm{g}$ & Cl $\%$ & AgCl $\mathrm{g}$ & $\mathrm{Cl} \%$ & \\
\hline 0.2128 & 0 & 0.2798 & 32.51 & 0.0652 & 7.57 & 40.08 \\
\hline 0.1996 & 0.04 & 0.2470 & 30.60 & 0.0764 & 9.46 & 40.06 \\
\hline 0.2154 & 0.07 & 0.2568 & 29.48 & 0.0918 & 10.54 & 40.02 \\
\hline 0.2092 & 0.12 & 0.2462 & 29.10 & 0.0926 & 10.94 & 40.04 \\
\hline 0.2146 & 0.16 & 0.2296 & 26.45 & 0.1174 & 13.52 & 39.97 \\
\hline
\end{tabular}

1. c. S. 1599. 
Tabelle $9 \mathrm{~b}$. (Fortsetzung.)

\begin{tabular}{|c|c|c|c|c|c|c|}
\hline \multirow{2}{*}{$\begin{array}{c}\mathrm{CrCl}_{3} \cdot 6 \mathrm{H}_{9} \mathrm{O} \\
\mathrm{g}\end{array}$} & \multirow{2}{*}{$\begin{array}{c}\mathrm{HNO}_{3} \text { in Mol. } \\
\text { auf } 1 \mathrm{Mol} \text {. } \\
\mathrm{CrCl}_{8} .6 \mathrm{H}_{2} \mathrm{O}\end{array}$} & \multicolumn{2}{|c|}{ Sofort gefällt } & \multicolumn{2}{|c|}{$\begin{array}{c}\text { Im Filtrat } \\
\text { ausgeschieden }\end{array}$} & \multirow{2}{*}{$\begin{array}{l}\text { Gesamt- } \\
\text { eblor } \\
\text { in } \%\end{array}$} \\
\hline & & $\mathrm{AgCl} \mathrm{g}$ & $\mathrm{Cl} \%$ & $\mathrm{AgCl} \mathrm{g}$ & $\mathrm{Cl} \%$ & \\
\hline 0.2090 & 0.25 & 0.2222 & 26.29 & 0.1158 & 13.70 & 39.99 \\
\hline 0.2090 & 0.33 & 0.2024 & 23.95 & 0.1348 & 15.95 & 39.90 \\
\hline 0.2014 & 0.42 & 0.1776 & 21.80 & 0.1476 & 18.12 & 39.92 \\
\hline 0.2142 & 0.8 & 0.1644 & 18.98 & 0.1818 & 20.98 & 39.96 \\
\hline 0.1992 & 1.7 & 0.1316 & 16.34 & 0.1904 & 23.63 & 39.97 \\
\hline 0.2100 & 2.4 & 0.1316 & 15.50 & 0.2068 & 24.35 & 39.85 \\
\hline 0.1996 & 3.4 & 01216 & 15.06 & 0.1994 & 24.70 & 39.76 \\
\hline 0.2186 & 3.9 & 0.1298 & 14.68 & 0.2230 & 25.22 & 39.90 \\
\hline 0.2036 & 5.9 & 0.1196 & 14.53 & 0.2092 & 25.40 & 39.93 \\
\hline 0.2176 & 7.1 & 0.1272 & 14.45 & 0.2246 & 25.52 & 39.97 \\
\hline 0.2106 & 10.2 & 0.1204 & 14.14 & 0.2198 & 25.81 & 39.95 \\
\hline 0.2104 & 15.5 & 0.1226 & 14.41 & 0.2174 & 25.55 & 39.96 \\
\hline 0.2092 & 23.4 & 0.1228 & 14.51 & 0.2162 & 25.55 & 40.06 \\
\hline 0.2026 & 40.2 & 0.1210 & 14.77 & 0.2070 & 25.26 & 40.03 \\
\hline 0.1930 & 59 & 0.1146 & 14.68 & 0.1974 & 25.29 & 39.97 \\
\hline 0.1892 & 86.1 & 0.1152 & 15.05 & 0.1906 & 24.91 & 39.96 \\
\hline 0.2088 & 117 & 0.1286 & 15.23 & 0.2084 & 24.68 & 39.91 \\
\hline 0.1980 & 165 & 0.1252 & 15.63 & 0.1956 & 24.42 & 40.05 \\
\hline 0.2120 & 231 & 0.1372 & 16.00 & 0.2054 & 23.96 & 39.96 \\
\hline 0.1972 & 317 & 0.1610 & 20.19 & 0.1584 & 19.86 & 40.05 \\
\hline 0.2072 & 402 & 0.1774 & 21.17 & 0.1564 & 18.66 & 39.83 \\
\hline 0.2000 & 520 & 0.1812 & 22.40 & 0.1424 & 17.60 & 40.00 \\
\hline 0.2156 & 579 & 0.2000 & 22.94 & 0.1492 & 17.11 & 40.05 \\
\hline 0.1950 & 976 & 0.2912 & 36.93 & 0.0240 & 3.04 & 39.97 \\
\hline
\end{tabular}

Wiederum drückt die Säure die Menge des sogleich gefällten Chlors stark herab. Nur werden dieselben Chlorwerte erst bei Zusatz ron etwas mehr Salpetersäure erreicht, als bei $0^{\circ}$. $2 / 3$ des vorhandenen Chlors fallen bei Gegenwart von etwa $1 / 6$ Mol. Salpetersäure (bei $0^{0}$ von $1 / 8 \mathrm{Mol}$.), $1 / 3$ fällt bei $10 \mathrm{Mol}$. (bei $0^{0}$ bei $6 \mathrm{Mol}$ ), sodann steigt bei Steigerung der Säure das gefällte Chlor etwas rascher als bei $0^{\circ}$, erreicht aber auch bei 5:9 Mol. noch nicht ${ }^{2} / 3$.

WeRNer und GUBser fanden bei einem Versuch bei gewöhnlicher Temperatur, der aber sonst wie der oben zitierte ausgeführt wurde $\left(0.4 \mathrm{~g} \mathrm{CrCl}_{3} .6 \mathrm{H}_{2} \mathrm{O}, 1.0 \mathrm{~g}_{\mathrm{AgNO}_{3}}, 15\right.$ Tropfen konzentrierte $\mathrm{NO}_{3} \mathrm{H}=$ etwa $0.7 \mathrm{~g} \mathrm{HNO}_{3}$ ) $24.67 \%$ Chlor. Bei ähnlichen Mengenverhältnissen von Chromchlorid, Silbernitrat und Salpetersäure erhielten wir das folgende Resultat: 


\begin{tabular}{|c|c|c|c|c|c|}
\hline $\begin{array}{l}\mathrm{CrCl}_{3} \cdot 6 \mathrm{H}_{2} \mathrm{O} \\
\mathrm{g}\end{array}$ & $\begin{array}{c}\mathrm{AgNO}_{3} \\
\mathrm{~g}\end{array}$ & $\begin{array}{l}\mathrm{HNO}_{3} \\
\mathrm{~g}\end{array}$ & $\begin{array}{l}\mathrm{AgCl} \text { sogleich } \\
\text { gefällt }\end{array}$ & $\begin{array}{l}\mathrm{AgCl} \text { aus d. Filtrat } \\
\text { abgeschieden }\end{array}$ & $\begin{array}{l}\% \mathrm{Cl} \mathrm{im} \\
\text { Gamzen }\end{array}$ \\
\hline 0.2030 & 0.5 & 0.28 & $0.1216 \mathrm{~g}=14.8 \% \mathrm{Cl}$ & $0.2074 \mathrm{~g}=25.3 \% \mathrm{Cl}$ & 40.1 \\
\hline 0.2018 & 0.5 & 0.51 & $0.1146 \mathrm{~g}=14.0 \% \mathrm{Cl}$ & $0.2116 \mathrm{~g}=25.9 \% \mathrm{Cl}$ & 39.9 \\
\hline
\end{tabular}

Statt der von WERNER annähernd gefundenen $2 / 3$ fanden wir somit nur $1 / 3$ des Chlors. Auch aus der Tabelle $9 \mathrm{~b}$ ist ersichtlich, dafs bei diesem Mengenverhältnis 14-15\% Chlor erhalten werden. Die von WERnER und GUBSER beobachteten $2 / 3$ erhielten wir bei Gegenwart von viel weniger Salpetersäure, nämlich 0.16 bis 0.33 Mol., statt der von Wenner und GUBser verwendeten 7 (s. o. Tabelle $9 \mathrm{~b}$ ). Auch folgende Versuche bestätigen dies, bei denen, wie von Werner und Gubser, etwa $4 \mathrm{Mol}$. Silbernitrat verwendet wurden, statt 3 wie in der Tabelle:

\begin{tabular}{c|c|c|c|c|c}
\hline $\begin{array}{c}\mathrm{CrCl}_{3} .6 \mathrm{H}_{2} \mathrm{O} \\
\mathrm{g}\end{array}$ & $\begin{array}{c}\mathrm{AgNO} \\
\mathrm{g}\end{array}$ & $\begin{array}{c}\mathrm{HNO}_{\mathrm{B}} \\
\mathrm{g}\end{array}$ & $\begin{array}{c}\mathrm{AgCl} \text { sogleich } \\
\text { gefällt }\end{array}$ & $\begin{array}{c}\text { AgCl aus d. Filtrat } \\
\text { abgeschieden }\end{array}$ & $\begin{array}{c}\% \text { Cl im } \\
\text { Ganzen }\end{array}$ \\
\hline \hline 0.2114 & 0.5 & 0.01 & $0.2408 \mathrm{~g}=28.2 \% \mathrm{Cl}$ & $0.1024 \mathrm{~g}=12.0 \% \mathrm{Cl}$ & 40.2 \\
0.1900 & 0.5 & 0.02 & $0.1736 \mathrm{~g}=22.5 \% \mathrm{Cl}$ & $0.1344 \mathrm{~g}=17.5 \% \mathrm{Cl}$ & $\mathbf{4 0 . 0}$ \\
\multicolumn{5}{|c}{ Gesamtflüssigkeit $30 \mathrm{ccm}}$.
\end{tabular}

PeLigot und später Jörgensen fanden wie oben erwähnt (S. 296), dafs durch Silbernitrat $2 / 3$ des Chlors gefällt werden. Sie haben bei gewöhnlicher Temperatur gearbeitet. Ob sie den üblichen Salpetersäurezusatz gemacht haben, geben sie nicht an, wir halten es aber nach den Ergebnissen unserer Versuche für wahrscheinlich, da wir mit Silbernitrat allein stets mehr als $2 / 3$ erbalten haben (siehe Tabelle $1 \mathrm{a}$, S. 299). PeLigor sagt, dafs er das Verfahren der Chlorbestimmung von PeLouze, nach welchem mit einer Lösung einer bestimmten Menge Silber in Salpetersäure titriert wird, angewendet habe. Hiernach ist es möglich, dafs der zur Lösung des Silbers benützte Überschufs von Salpetersäure nicht völlig vertrieben worden war, dafs die Lösung also schwach salpetersauer war. Das würde dann mit unseren Versuchen übereinstimmen, nach welchen $2 / 3$ des Chlors gefällt werden, wenn die Lösung auf 1 Mol. Chromchlorid 0.16-0.25 Mol. Salpetersäure enthält.

JörgEnsen macht keine Angaben über die Art der Fällung.

Die in der folgenden Tabelle $9 \mathrm{c}$ angegebenen Versuche zeigen z. anorg. Chem. Bd. 39 . 
den Einflufs vermehrten Silbernitrąts bei gleichbleibender Säuremenge; von dieser setzten wir 6 Mol. zu, bei welcher Menge nach den Versuchen der Tabelle 9 a $1 / 3$ des vorhandenen Chlors gefällt wird.

\section{Tabolle $9 \mathrm{c}$.}

Chlorfällungen im grünen Chromchloridhydrat durch steigende Mengen Silbernitrat unter Zusatz von 6 Mol. Sulpetersäure. Temperatur $0^{\circ}$. Gesamtvolumen $25 \mathrm{ccm}$ auf 0.1 Chromchloridhydrat. Erste Fällung sofort abfiltriert.

\begin{tabular}{|c|c|c|c|c|c|c|}
\hline \multirow{2}{*}{$\begin{array}{c}\mathrm{CrCl}_{3} \cdot 6 \mathrm{H}_{2} \mathrm{O} \\
\mathrm{g}\end{array}$} & \multirow{2}{*}{$\begin{array}{l}\text { AgNO } \mathrm{NO}_{3} \text { be- } \\
\text { rechnet für } \\
\text { Atome Cblor }\end{array}$} & \multicolumn{2}{|c|}{ Sofort gefällt } & \multicolumn{2}{|c|}{$\begin{array}{c}\text { Im Filtrat } \\
\text { ausgeschieden }\end{array}$} & \multirow{2}{*}{$\begin{array}{l}\text { Gesamt- } \\
\text { ehlor } \\
\text { in } \%\end{array}$} \\
\hline & & $\mathrm{AgCl} \mathrm{g}$ & $\mathrm{Cl} \%$ & $\mathrm{AgCl} g$ & $\mathrm{Cl} \%$ & \\
\hline 0.1000 & 3 & 0.0567 & 14.02 & 0.1046 & 25.86 & 39.88 \\
\hline 0.1036 & 6 & 0.0594 & 14.18 & 0.1076 & 25.68 & 39.86 \\
\hline 0.1046 & 12 & 0.0600 & 14.19 & 0.1078 & 25.48 & 39.67 \\
\hline 0.1016 & 24 & 0.0598 & 14.55 & 0.1042 & 25.36 & 39.91 \\
\hline 0.1005 & 36 & 0.0596 & 14.67 & 0.1020 & 25.09 & 39.76 \\
\hline 0.1106 & 48 & 0.0734 & 16.41 & 0.1062 & 23.74 & 40.15 \\
\hline
\end{tabular}

Aus diesen Versuchen ergibt sich, dafs erst bei grofser Steigerung des Silbernitrats die Menge des sogleich gefällten Chlors deutlich zunimmt, sie beträgt bei $48 \mathrm{Mol} .16 .4 \%$. Indessen kann diese Wirkung des gesteigerten Silbernitrats, mehr Chlor zu fällen, durch Zusatz von mehr Salpetersäure wieder aufgehoben werden, man erreicht mit anderen Worten bei Fällungen mit mehr Silbernitrat das Minimum des gefällten Chlors erst bei Zusatz von mehr Salpetersänre. Bei den in der folgenden Tabelle geschilderten Versuchen wurde mit $9 \mathrm{Mol}$. Silbernitrat gefällt.

(S. Tabelle $9 \mathrm{~d}, \mathrm{~S} .311$.)

Vergleicht man diese Werte mit denen der Tabelle 9 a (S. 306), so siebt man, dafs bei derselben Menge Salpetersäure zunächst immer mehr Chlor erhalten wird. Aber bei etwa $12 \mathrm{Mol}$. ist $1 / 3$ erreicht, (bei $3 \mathrm{Mol}$. Silbernitrat: $6 \mathrm{Mol}$. Salpetersäure); von etwa 100 Mol. Salpetersäure an vergrölsert sich dann die Menge des gefällten Chlors wieder, aber zunächst langsamer als bei den Fällungen mit $3 \mathrm{Mol}$. Silbernitrat. Um dies anschaulich zu machen haben wir in der Tabelle $9 \mathrm{~d}$ in der letzten Spalte diejenigen Mengen Chlor zusammengestellt, welche bei Fällungen mit 3 Mol. Silbernitrat bei Gegenwart derselben Mengen Salpetersäure erhalten werden (die Werte sind aus der Tabelle 9 a berechnet). 
Tabelle $9 \mathrm{~d}$.

Chlorfällungen im grünen Chromchlorhydrat mit für 9 Atome Chlor berechnetem Silbernitrat unter Zusatz steigender Mengen Salpetersäure. Temperatur $0^{\circ}$. Gesamtvolumen $25 \mathrm{ccm}$. Erste Fällung sofort abfiltriert.

\begin{tabular}{|c|c|c|c|c|c|c|c|}
\hline $\begin{array}{c}\mathrm{CrCl}_{8} . \\
6 \mathrm{H}_{2} \mathrm{O} \\
\mathrm{g}\end{array}$ & $\begin{array}{l}\mathrm{HNO}_{3} \\
\text { in } \mathrm{Mol} \text {. }\end{array}$ & $\begin{array}{l}\text { Sofort g } \\
\mathrm{AgCl} \mathrm{g}\end{array}$ & Clällt & $\begin{array}{l}\operatorname{Im} \mathrm{Fi} \\
\text { ausgesch } \\
\mathrm{AgCl} \mathrm{g}\end{array}$ & $\begin{array}{l}\text { trat } \\
\text { ieden } \\
\mathrm{Cl} \%\end{array}$ & $\begin{array}{l}\text { Gesamt- } \\
\text { chlor- } \\
\text { in } \%\end{array}$ & $\begin{array}{l}\% \mathrm{Cl} \text { sofort } \\
\text { gefällt durch } \\
3 \mathrm{Mol} . \mathrm{AgNO}\end{array}$ \\
\hline 0.1088 & 0 & 0.1600 & 36.36 & 0.0160 & 3.63 & 39.99 & 31.56 \\
\hline 0.1036 & 0.16 & 0.1232 & 29.40 & 0.0436 & 10.41 & 39.81 & 25.0 \\
\hline 0.1006 & 0.68 & 0.0773 & 19.00 & 0.0844 & 20.75 & 39.7 & 17.5 \\
\hline 0.1092 & 1.18 & 0.0764 & 17.30 & 0.1000 & 22.64 & 39.94 & 16.4 \\
\hline 0.1054 & 4.8 & 0.0616 & 14.45 & 0.1084 & 25.43 & 39.88 & 14.3 \\
\hline 0.1030 & 12.5 & 0.0588 & 14.11 & 0.1072 & 25.73 & 39.84 & 14.0 \\
\hline 0.1014 & 25.3 & 0.0570 & 13.90 & 0.1062 & 25.90 & 39.80 & 14.3 \\
\hline 0.1016 & 49.2 & 0.0578 & 14.07 & 0.1058 & 25.75 & 39.82 & 14.5 \\
\hline 0.1006 & t24 & 0.0594 & 14.60 & 0.1030 & 25.32 & 39.92 & 15.1 \\
\hline 0.1072 & 194 & 0.0646 & 14.90 & 0.1076 & 24.82 & 39.72 & 15.5 \\
\hline 0.1050 & 278 & 0.0658 & 15.49 & 0.1028 & 24.21 & 39.70 & 16.6 \\
\hline 0.1058 & 354 & 0.0664 & 15.52 & 0.1040 & 24.30 & 39.82 & 17.7 \\
\hline 0.1036 & 504 & 0.0818 & 19.52 & 0.0854 & 20.38 & 39.90 & 20.7 \\
\hline 0.1058 & 590 & 0.1162 & 27.16 & 0.0540 & 12.62 & 39.78 & 22.5 \\
\hline 0.1054 & 988 & 0.1455 & 34.13 & 0.0234 & 5.49 & 39.92 & - \\
\hline
\end{tabular}

Dals die Verdünnung der Lösungen keinen Einflufs auf die gefällten Chlorsilbermengen hat, zeigen die folgenden $\nabla$ ersuche.

\section{Tabelle $9 \mathrm{e}$.}

Chlorfällungen im grünen Chromchloridhydrat mit für 3 Atome Chlor berechnetem Silbernitrat unter Zusatz von je 6 Mol. Salpetersäure bei steigendem Volumen der Gesamtlüssigkeit. Temperatur $0^{0}$. Die erste Fällung wurde sofort abfiltriert.

\begin{tabular}{|c|c|c|c|c|c|c|}
\hline \multirow{2}{*}{$\begin{array}{c}\mathrm{CrCl}_{3} \cdot 6 \mathrm{H}_{2} \mathrm{O} \\
\mathrm{g}\end{array}$} & \multirow{2}{*}{$\begin{array}{l}\text { Gesamtflïs- } \\
\text { sigkeitsvolu- } \\
\text { men in ccm }\end{array}$} & \multicolumn{2}{|c|}{ Sofort gefällt } & \multicolumn{2}{|c|}{$\begin{array}{c}\text { Im Filtrat } \\
\text { abgeschieden }\end{array}$} & \multirow{2}{*}{$\begin{array}{l}\text { Gesamt- } \\
\text { chlor } \\
\text { in } \%\end{array}$} \\
\hline & & $\mathrm{AgCl} g$ & $\mathrm{Cl} \%$ & $\mathrm{AgCl} \mathrm{g}$ & Cl $\%$ & \\
\hline 0.2038 & 25 & 0.1154 & 14.00 & 0.2128 & 25.82 & 39.82 \\
\hline 0.2000 & 50 & 0.1134 & 14.02 & 0.2092 & 25.86 & 39.88 \\
\hline 0.2052 & 100 & 0.1170 & 14.10 & 0.2134 & 25.71 & 39.81 \\
\hline 0.2034 & 200 & 0.1156 & 14.05 & 0.2114 & 25.70 & 39.75 \\
\hline 0.2080 & 250 & 0.1168 & 13.90 & 0.2186 & 25.99 & 39.89 \\
\hline
\end{tabular}


Schliefslich seien noch die folgenden Versuche angeführt, bei denen erst nach Verlauf einiger Zeit abfiltriert wurde.

\section{Tabelle $9 \mathrm{f}$.}

Chlorfällungen im grünen Chromchloridhydrat mit für 3 Atome Chlor berechnetem Silbernitrat unter Zusatz von je 3 Mol. Salpetersäure auf 1 Mol. Chromchlorid. Das gefällte Chlorsilber wird erst nach einiger Zeit abfiltriert. Temperatur $0^{\circ}$. Gesamtvolumen $50 \mathrm{ccm}$.

\begin{tabular}{|c|c|c|c|c|c|c|}
\hline \multirow{2}{*}{$\begin{array}{c}\mathrm{CrCl}_{3} \cdot 6 \mathrm{H}_{2} \mathrm{O} \\
\mathrm{g}\end{array}$} & \multirow{2}{*}{\begin{tabular}{|c|} 
Zeit des \\
Stehenlassens \\
vor dem \\
Abfiltrieren \\
\end{tabular}} & \multicolumn{2}{|c|}{ Erste Fällung } & \multicolumn{2}{|c|}{$\begin{array}{c}\text { Im Filtrat } \\
\text { abgeschieden }\end{array}$} & \multirow{2}{*}{$\begin{array}{l}\text { Gesamt- } \\
\text { chlor } \\
\text { in } \%\end{array}$} \\
\hline & & $\mathrm{AgCl} \mathrm{g}$ & $\mathrm{Cl} \%$ & $\mathrm{AgCl} \mathrm{g}$ & $\mathrm{Cl} \%$ & \\
\hline 0.1964 & sofort abfiltr. & 0.1142 & 14.38 & 0.2032 & 25.58 & 39.96 \\
\hline 0.2048 & 2 Min. & 0.1216 & 14.68 & 0.2086 & 25.18 & 39.86 \\
\hline 0.2016 & $5 \%$ & 0.1220 & 14.96 & 0.2034 & 24.95 & 39.91 \\
\hline 0.2072 & 10 & 0.1296 & 15.47 & 0.2048 & 24.44 & 39.91 \\
\hline 0.2058 & 15 & 0.1336 & 16.05 & 0.1990 & 23.91 & 39.96 \\
\hline 0.2062 & 20 & 0.1364 & 16.36 & 0.1970 & 23.62 & 39.98 \\
\hline 0.2014 & 30 & 0.1386 & 17.02 & 0.1870 & 22.96 & 39.98 \\
\hline
\end{tabular}

Wie man sieht, steigt die Menge des gefällten Chlorsilbers langsam mit der Zeit, aber selbst wenn man erst nach 10 Minuten filtriert, findet man nur $1.1 \%$ Chlor mehr, als wenn man sogleich filtriert, nach 30 Minuten $2.6 \%$ mehr.

10. Mit Silbersulfat unter Zusatz von Schwefelsäure.

Auch hier zeigt sich, wie aus der folgenden Tabelle 10 hervorgeht, deutlich die Wirkung des Säurezusatzes.

Tabelle 10.

Chlorfällungen im grünen Chromchloridhydrat mit für 3 Atome Chlor berechnetem Silbersulfat unter Zusatz steigender Mengen Schwefelsäure bei $0^{\circ}$ und $60 \mathrm{~cm}$ Gesamtvolumen. Erste Fallung sofort abfiltriert.

\begin{tabular}{|c|c|c|c|c|c|c|}
\hline \multirow{2}{*}{$\begin{array}{c}\mathrm{CrCl}_{3} \cdot 6 \mathrm{H}_{2} \mathrm{O} \\
\mathrm{g}\end{array}$} & \multirow{2}{*}{$\mid \begin{array}{c}\mathrm{H}_{2} \mathrm{SO}_{4} \text { in } \mathrm{Mol} . \\
\text { auf je } 1 \mathrm{Mol} . \\
\mathrm{CrCl}_{3} .6 \mathrm{H}_{2} \mathrm{O}\end{array}$} & \multicolumn{2}{|c|}{ Sofort gefallt } & \multicolumn{2}{|c|}{$\begin{array}{c}\text { Im Filtrat } \\
\text { abgeschieden }\end{array}$} & \multirow{2}{*}{$\begin{array}{c}\text { Gesamt- } \\
\text { chlor } \\
\text { in } \%\end{array}$} \\
\hline & & $\mathrm{AgCl} g$ & $\mathrm{Cl} \%$ & $\mathrm{AgCl} \mathrm{g}$ & $\mathrm{Cl} \%$ & \\
\hline 0.1052 & 0 & 0.1572 & 36.95 & 0.0132 & 3.10 & 40.05 \\
\hline 0.1026 & 0.14 & 0.1194 & 28.77 & 0.0462 & 11.13 & 39.90 \\
\hline 0.1120 & 0.27 & 0.1206 & 26.62 & 0.0600 & 13.25 & 39.87 \\
\hline 0.1062 & 0.54 & 0.0914 & 21.28 & 0.0798 & 18.58 & 39.86 \\
\hline 0.1014 & 1.1 & 0.0780 & 19.02 & 0.0852 & 20.78 & 39.80 \\
\hline 0.1102 & 2.1 & 0.0776 & 17.41 & 0.1002 & 22.48 & 39.89 \\
\hline 0.1012 & 8.6 & 0.0700 & 17.10 & 0.0938 & 22.92 & 40.02 \\
\hline 0.1074 & 16.2 & 0.0674 & 15.52 & 0.1064 & 24.49 & 40.01 \\
\hline
\end{tabular}


Tabelle 10 (Fortsetzung).

\begin{tabular}{|c|c|c|c|c|c|c|}
\hline \multirow{2}{*}{$\underset{\mathrm{g}}{\mathrm{CrCl}_{3} \cdot 6 \mathrm{H}_{2} \mathrm{O}}$} & \multirow{2}{*}{$\begin{array}{l}\mathrm{H}_{8} \mathrm{SO}_{4} \text { in } \mathrm{Mol} \\
\text { auf je } 1 \mathrm{Mol} \\
\mathrm{CrCl}_{3} .6 \mathrm{H}_{2} \mathrm{O}\end{array}$} & \multicolumn{2}{|c|}{ Sofort gefällt } & \multicolumn{2}{|c|}{$\begin{array}{c}\text { Im Filtrat } \\
\text { abgeschieden }\end{array}$} & \multirow{2}{*}{$\begin{array}{c}\text { Gesamt } \\
\text { chlor } \\
\text { in } \%\end{array}$} \\
\hline & & $\mathrm{AgCl} \mathrm{g}$ & $\mathrm{Cl} \%$ & $\mathrm{AgCl} \mathrm{g}$ & $\mathrm{Cl} \%$ & \\
\hline 0.1056 & 33.1 & 0.0652 & 15.27 & 0.1052 & 24.63 & 39.90 \\
\hline 0.1056 & 66.1 & 0.0646 & 15.13 & 0.1062 & 24.87 & 40.00 \\
\hline 0.1030 & 136 & 0.0636 & 15.27 & 0.1032 & 24.77 & 40.04 \\
\hline 0.1070 & 261 & 0.0726 & 16.78 & 0.1006 & 23.25 & 40.03 \\
\hline 0.1040 & 403 & 0.0718 & 17.07 & 0.0962 & 22.87 & 39.94 \\
\hline 0.1018 & 549 & 0.0790 & 19.19 & 0.0852 & 20.69 & 39.88 \\
\hline 0.1082 & 986 & 0.1292 & 29.52 & 0.0462 & 10.56 & 40.08 \\
\hline
\end{tabular}

Während ohne Zusatz von Säure direkt etwa ${ }^{12} / 1$ des vorhandenen Chlors ausfallen, hat Zusatz von etwa $1 / 4$ Mol. Schwefelsäure auf $1 \mathrm{Mol}$. Chromchloridhydrat die Folge, dals nur $2 / 3$ niedergeschlagen werden. Weiterhin nimmt das Chlor bei gesteigerter Schwefelsäure stetig ab, sinkt aber nicht wie bei der Salpetersäure bis auf $1 / 3(13.3 \%)$ herab, sondern es bleibt bei etwa $15 \%$ stehen. Diese Menge wird gefällt bei Gegenwart von 16-136 Mol. Schwefelsäure. Vermehrt man die Schwefelsäure noch mehr, so wird wieder mehr gefällt, aber auch bei Zusatz sehr grofser Mengen werden nicht viel mehr als $2 / 3$ des vorhandenen Chlors niedergeschlagen.

11. Mit Silberchlorat unter Zusatz von Chlorsäure.

Tabelle 11.

Chlorfallungen im grünen Chromehloridhydrat mit für 3 Atome Chlor berechnetem Silberchlorat unter Zusatz steigender Mengen Chlorsäure bei $0^{\circ}$ und $50 \mathrm{ccm}$ Gesamtvolumen. Erste Fällung sofort abfiltriert.

\begin{tabular}{|c|c|c|c|c|c|c|}
\hline \multirow{2}{*}{$\begin{array}{c}\mathrm{CrCl}_{3}, 6 \mathrm{H}_{2} \mathrm{O} \\
\mathrm{g}\end{array}$} & \multirow{2}{*}{$\left|\begin{array}{c}\mathrm{HClO}_{3} \text { in } \mathrm{Mol} . \\
\text { auf } 1 \mathrm{Mol} . \\
\mathrm{CrCl}_{8} \cdot 6 \mathrm{H}_{2} \mathrm{O}\end{array}\right|$} & \multicolumn{2}{|c|}{ Sofort gefällt } & \multicolumn{2}{|c|}{$\begin{array}{c}\text { Im Filtrat } \\
\text { abgeschieden }\end{array}$} & \multirow{2}{*}{$\begin{array}{c}\text { Gesamt- } \\
\text { chlor } \\
\text { in } \%\end{array}$} \\
\hline & & $\mathrm{AgCl} \mathrm{g}$ & $\mathrm{Cl} \%$ & $\mathrm{AgCl} \mathrm{g}$ & $\mathrm{Cl} \%$ & \\
\hline 0.2008 & 0 & 0.2514 & 30.95 & 0.0728 & 8.96 & 39.91 \\
\hline 0.2024 & 0.02 & 0.2344 & 28.63 & 0.0926 & 11.31 & 39.94 \\
\hline 0.2048 & 0.08 & 0.1712 & 20.67 & 0.1608 & 19.41 & 40.08 \\
\hline 0.2016 & 0.2 & 0.1512 & 18.55 & 0.1740 & 12.34 & 39.89 \\
\hline 0.2034 & 0.42 & 0.1332 & 16.19 & 0.1942 & 23.61 & 39.80 \\
\hline 0.2064 & 0.81 & 0.1272 & 15.24 & 0.2086 & 24.99 & 40.23 \\
\hline 0.2026 & 1.45 & 0.1178 & 14.38 & 0.2098 & 25.60 & 39.98 \\
\hline 0.2082 & 2 & 0.1180 & 14.01 & 0.2190 & 26.01 & 40.02 \\
\hline 0.2074 & 4 & 0.1156 & 13.78 & 0.2198 & 26.20 & 39.98 \\
\hline
\end{tabular}


Tabelle 11 (Fortsetzung).

\begin{tabular}{|c|c|c|c|c|c|c|}
\hline \multirow{2}{*}{$\begin{array}{c}\mathrm{CrCl}_{3} \cdot 6 \mathrm{H}_{2} \mathrm{O} \\
\mathrm{g}\end{array}$} & \multirow{2}{*}{$\begin{array}{c}\mathrm{HClO}_{3} \text { in } \mathrm{Mol} . \\
\text { auf je } 1 \mathrm{Mol} . \\
\mathrm{CrCl}_{3} .6 \mathrm{H}_{2} \mathrm{O}\end{array}$} & \multicolumn{2}{|c|}{ Sofort gefällt } & \multicolumn{2}{|c|}{$\begin{array}{c}\text { Im Filtrat } \\
\text { abgeschieden }\end{array}$} & \multirow{2}{*}{$\begin{array}{l}\text { Gesamt- } \\
\text { ehlor } \\
\text { in } \%\end{array}$} \\
\hline & & $\mathrm{AgCl} g$ & Cl $\%$ & $\mathrm{AgCl} g$ & Cl $\%$ & \\
\hline 0.2016 & 6.2 & 0.1116 & 13.69 & 0.2144 & 26.30 & 39.99 \\
\hline 0.2032 & 8.3 & 0.1120 & 13.63 & 0.2160 & 26.28 & 39.91 \\
\hline 0.2034 & 10.5 & 0.1120 & 13.62 & 0.2186 & 26.57 & 40.19 \\
\hline 0.2086 & 14.1 & 0.1152 & 13.66 & 0.2214 & 26.24 & 39.90 \\
\hline 0.2052 & 20.4 & 0.1140 & 13.74 & 0.2202 & 26.53 & $40.27^{1}$ \\
\hline 0.2064 & 40.7 & 0.1150 & 13.78 & 0.2212 & 26.50 & 40.28 \\
\hline 0.2044 & 61.6 & 0.1138 & 13.77 & 0.2210 & 26.73 & 40.50 \\
\hline 0.2068 & 81.2 & 0.1150 & 13.75 & 0.2240 & 26.78 & 40.53 \\
\hline 0.2066 & 102 & 0.1144 & 13.69 & 0.2314 & 27.69 & 41.38 \\
\hline 0.2098 & 137 & 0.1192 & 14.05 & 0.2312 & 27.25 & 41.30 \\
\hline 0.2034 & 211 & 0.1178 & 14.32 & 0.2232 & 27.13 & 41.45 \\
\hline 0.2072 & 242 & 0.1226 & 14.63 & 0.2290 & 27.33 & 41.96 \\
\hline 0.1920 & 500 & 0.1040 & 13.39 & 0.2188 & 28.18 & 41.57 \\
\hline
\end{tabular}

Der Einflufs des Chlorsäurezusatzes auf die gefällten Chlorsilbermengen gleicht im allgemeinen jenem der Salpetersäure (Tabelle 9 a, S. 306), nur mit dem Unterschied, dafs dieselbe Chlormenge schon bei Gegenwart von viel weniger Chlorsäure erhalten wird, als von Salpetersäure, und dals infolgedessen das Minimum von $1 / 3$ rascher erreicht wird: Bei Gegenwart von etwa $1 / 20 \mathrm{Mol}$. Chlorsäure werden $2 / 3$ gefällt (bei der Salpetersäure von etwa $1 / 8 \mathrm{Mol}$.); $1 / 3$ erhält man schon bei Gegenwart von etwa $3 \mathrm{Mol}$., bei der Salpetersäure von 6 . Weiterer Zusatz von Chlorsäure verändert die Menge des niedergeschlagenen Chlors nicht wesentlich, im Gegensatz zur Salpetersäure, bei deren vermehrtem Zusatz das Chlor sich schon bei $50 \mathrm{Mol}$. wieder zu heben beginnt und von da an ziemlich stark zunimmt.

12. Mit Silberperchlorat unter Zusatz von Überchlorsäure.

(S. Tabelle 12, S. 315.)

Die Überchlorsäure verhält sich der Chlorsäure sehr ähnlich; bei Zusatz von etwa $1 / 20$ Mol. werden $2 / 3$, bei Zusatz von $3 \mathrm{Mol} .1 / 3$ des Chlors gefällt. Auch durch Steigerung der Überchlorsäure bis auf $500 \mathrm{Mol}$. wird, wie bei der Chlorsäure, nicht mehr Chlor niedergeschlagen. Hiermit in Übereinstimmung, aber im Gegensatz

${ }^{1}$ Das Gesantchlor ist bei diesem und den folgenden Versuchen aus den in der Tabelle 3 (S. 302) angegebenen Gründen zu hoch. 


\section{Tabelle 12.}

Chlorfällungen im grünen Chromchloridhydrat mit für 3 Atome Chlor berechnetem Silberperchlorat unter Zusatz steigender Mengen Überchlorsäure. Temperatur $0^{\circ}$. Gesamtvolimen $25 \mathrm{ccm}$. Erste Fïllung sofort abfiltriert.

\begin{tabular}{|c|c|c|c|c|c|c|}
\hline \multirow{2}{*}{$\begin{array}{c}\mathrm{CrCl}_{3} \cdot 6 \mathrm{H}_{2} \mathrm{O} \\
\mathrm{g}\end{array}$} & \multirow{2}{*}{$\begin{array}{l}\mathrm{HClO}_{4} \text { in } \mathrm{Mol} \text {. } \\
\text { auf je } 1 \mathrm{Mol} \text {. } \\
\mathrm{CrCl}_{3} .6 \mathrm{H}_{2} \mathrm{O}\end{array}$} & \multicolumn{2}{|c|}{ Sofort gefällt } & \multicolumn{2}{|c|}{$\begin{array}{c}\text { Im Filtrat } \\
\text { abgeschieden }\end{array}$} & \multirow{2}{*}{$\begin{array}{l}\text { Gesamt- } \\
\text { chlor } \\
\text { in } \%\end{array}$} \\
\hline & & $\mathrm{AgCl} \mathrm{g}$ & Cl $\%$ & $\mathrm{AgCl} g$ & Cl $\%$ & \\
\hline 0.0988 & 0 & 0.1148 & 28.73 & 0.0446 & 11.16 & 39.89 \\
\hline 0.1030 & 0.05 & 0.1120 & 26.89 & 0.0534 & 12.82 & 39.71 \\
\hline 0.1000 & 0.14 & 0.1068 & 26.41 & 0.0534 & 13.21 & 39.62 \\
\hline 0.1080 & 0.25 & 0.1104 & 25.27 & 0.0640 & 14.65 & 39.92 \\
\hline 0.1046 & 1.3 & 0.0744 & 17.59 & 0.0940 & 22.22 & 39.81 \\
\hline 0.1092 & 2.5 & 0.0622 & 14.09 & 0.1132 & 25.63 & 39.72 \\
\hline 0.1060 & 13.2 & 0.0588 & 13.72 & 0.1122 & 26.17 & 39.89 \\
\hline 0.1028 & 27.1 & 0.0568 & 13.66 & 0.1092 & 26.27 & 39.93 \\
\hline 0.1080 & 55 & 0.0592 & 13.55 & 0.1146 & 26.24 & 39.79 \\
\hline 0.1100 & 76.2 & 0.0614 & 13.80 & 0.1160 & 26.08 & 39.88 \\
\hline 0.1086 & 103 & 0,0624 & 14.21 & 0.1122 & 25.55 & 39.76 \\
\hline 0.1084 & 136 & 0.0592 & 13.50 & 0.1148 & 26.19 & 39.69 \\
\hline 0.1054 & 159 & 0.0592 & 13.89 & 0.1108 & 25.99 & 39.88 \\
\hline 0.1070 & 276 & 0.0596 & 13.77 & 0.1140 & 26.34 & 40.11 \\
\hline 0.1100 & 500 & 0.0604 & 13.58 & 0.1168 & 26.26 & 39.84 \\
\hline
\end{tabular}

zum Verhalten der andern Silbererze, hatte sich bei den Versuchen mit Silberperchlorat allein (s. Tabelle 4, S. 302) gezeigt, dals Steigerung desselben keine Vermehrung des gefällten Chlors zur Folge hatte, eher eine Verminderung.

13. Mit Silberpermanganat unter Zusatz von Übermangansäure.

Tabelle 13.

Chlorfällungen im grünen Chromchloridhydrat mit für 3 Atome Chlor berechnetem Silberpermanganat unter Zusatz steigender Mengen Übermangansäure. ${ }^{1}$ Temperatur $0^{0}$. Gesamtvolumen $100 \mathrm{~cm}$. Erste Fällung sofort abfiltriert.

\begin{tabular}{|c|c|c|c|c|c|c|}
\hline \multirow{2}{*}{$\begin{array}{c}\mathrm{CrCl}_{3} \cdot 6 \mathrm{H}_{2} \mathrm{O} \\
\mathrm{g}\end{array}$} & \multirow{2}{*}{$\begin{array}{c}\mathrm{HMnO}_{4} \\
\text { in } \mathrm{Mol} \text {. auf } \\
\text { je } 1 \mathrm{Mol} \text {. } \\
\mathrm{CrCl}_{3} .6 \mathrm{H}_{2} \mathrm{O}\end{array}$} & \multicolumn{2}{|c|}{ Sofort gefällt } & \multicolumn{2}{|c|}{$\begin{array}{c}\text { Im Filtrat } \\
\text { abgeschieden }\end{array}$} & \multirow{2}{*}{$\begin{array}{l}\text { Gesamt- } \\
\text { chlor } \\
\text { in } \%\end{array}$} \\
\hline & & $\mathrm{AgCl} g$ & $\mathrm{Cl} \%$ & $\mathrm{AgCl} \mathrm{g}$ & $\mathrm{Cl} \%$ & \\
\hline 0.1334 & 0 & 0.1566 & 29.03 & 0.0600 & 11.12 & 40.15 \\
\hline 0.1208 & 3 & 0.0712 & 14.57 & 0.1244 & 25.46 & 40.03 \\
\hline 0.1186 & 6 & 0.0682 & 14.22 & 0.1246 & 25.98 & 40.20 \\
\hline 0.0852 & 12 & 0.0470 & 13.64 & 0.0920 & 26.70 & 40.34 \\
\hline 0.1048 & 24 & 0.0600 & 14.16 & 0.1094 & 25.81 & 39.97 \\
\hline 0.0976 & 36 & 0.0570 & 14.44 & 0.1022 & 25.89 & 40.33 \\
\hline
\end{tabular}

1 Aus $\mathrm{Ba}\left(\mathrm{MnO}_{4}\right)_{2}$ mittels verdünnter Schwefelsäure in berechneter Menge bei $0^{\circ}$ dargestellt. Das $\mathrm{Ba}\left(\mathrm{MnO}_{4}\right)_{2}$ wurde durch Umsetzung von $\mathrm{AgMnO}_{4}$ mit $\mathrm{BaCl}$ erhalten. 
Auch diese Säure drückt die Menge des sogleich gefällten Chlors stark herunter, man erreicht wie bei der Salpetersäure mit $6 \mathrm{Mol}$., dafs nur $1 / 3$ des Chlors herausfällt. Steigerung der Übermangansäure bis auf $36 \mathrm{Mol}$. bewirkt keine Änderung.

14. Mit Silberlactat unter Zusatz von Milchsäure.

Tabelle 14.

Chlorfällungen im grünen Chromchloridhydrat mit für 3 Atome Chlor berechnetem Silberlaktat unter Zusatz steigender Mengen Milchsäure. Temperatur $0^{\circ}$. Gesamtvolumen $50 \mathrm{ccm}$. Erste Fällung sofort abfiltriert.

\begin{tabular}{|c|c|c|c|c|c|c|}
\hline \multirow{2}{*}{$\begin{array}{c}\mathrm{CrCl}_{3} \\
6 \mathrm{H}_{2} \mathrm{O} \\
\mathrm{g}\end{array}$} & \multirow{2}{*}{$\begin{array}{c}\text { Milchsäure in } \\
\text { Mol. auf } 1 \mathrm{Mol} \text {. } \\
\mathrm{CrCl}_{3} \cdot 6 \mathrm{H}_{2} \mathrm{O}\end{array}$} & \multicolumn{2}{|c|}{ Sofort gefällt } & \multicolumn{2}{|c|}{$\begin{array}{c}\text { Im Filtrat } \\
\text { abgeschieden }\end{array}$} & \multirow{2}{*}{$\begin{array}{l}\text { Gesamt- } \\
\text { chlor } \\
\text { in } \%\end{array}$} \\
\hline & & $\mathrm{AgCl} g$ & $\mathrm{Cl} \%$ & $\mathrm{AgCl} \mathbf{g}$ & $\mathrm{Cl} \%$ & \\
\hline 0.1070 & 0 & 0.1684 & 38.92 & 0.0034 & 0.79 & 39.71 \\
\hline 0.1126 & 1 & 0.1442 & 31.66 & 0.0368 & 8.08 & 39.74 \\
\hline 0.1034 & 3 & 0.1240 & 29.65 & 0.0426 & 10.19 & 39.84 \\
\hline 0.1042 & 15.1 & 0.1066 & 25.30 & 0.0612 & 14.52 & 39.82 \\
\hline 0.1040 & 30.2 & 0.0992 & 23.59 & 0.0680 & 16.17 & $39.7 b$ \\
\hline 0.1062 & 44.6 & 0.0758 & 17.65 & 0.0946 & 22.03 & 39.68 \\
\hline 0.1078 & 58.5 & 0.0688 & 15.78 & 0.1052 & 24.13 & 39.91 \\
\hline 0.1080 & 93 & 0.0676 & 15.48 & 0.1068 & 24.45 & 39.93 \\
\hline 0.1032 & 101 & 0.0634 & 15.19 & 0.1036 & 24.82 & 40.01 \\
\hline 0.1028 & 126 & 0.0646 & 15.54 & 0.1018 & 24.49 & 40.03 \\
\hline 0.1084 & 168 & 0.0690 & 15.74 & 0.1064 & 24.27 & 40.01 \\
\hline 0.1036 & 251 & 0.0692 & 16.52 & 0.0978 & 23.34 & 39.86 \\
\hline 0.1090 & 357 & 0.0802 & 18.19 & 0.0950 & 21.55 & 39.74 \\
\hline 0.1068 & 486 & 0.0894 & 20.70 & 0.0830 & 19.21 & 39.91 \\
\hline
\end{tabular}

Auch durch Zusatz dieser Säure wird die Menge des sogleich gefällten Chlors vermindert, aber man braucht von ihr weit grölsere Mengen, um dasselbe Chlor zu erhalten, als von den bisher bebehandelten Säuren. Von diesen letzteren war es die Schwefelsäure, von der am meisten zugesetzt werden mufste, um dieselbe Wirkung zu erzielen wie bei den anderen. $\mathrm{Um} 2 / 3$ des vorbandenen Chlors zu fällen, muss man etwa 9 Mol. Milchsäure auf 1 Mol. Chromchloridhydrat zusetzen (bei der Schwefelsäure etwa $1 / 4 \mathrm{Mol}$.). Fährt man mit der Steigerung des Milchsäurezusatzes fort, so kommt man bei etwa $100 \mathrm{Mol}$. auf ein Minimum von $15.2 \%$; bei der Schwefelsäure hatten wir dasselbe Minimum erreicht, aber schon bei Zusatz von $16 \mathrm{Mol}$. Bei Vermehrung dor Milchsäure erhält man wieder mehr Chlor, ähnlich wie bei der Schwefelsäure. 
15. Mit Silberacetat unter Zusatz von Essigsäure.

Tabelle $15 \mathrm{a}$.

Chlorfällungen im grünen Chromchloridhydrat mit für 3 Atome Chlor berechnetem Silberacetat unter Zusatz steigender Mengen Essigsaure. Temperatur $0^{\circ}$. Gesamtvolumen $50 \mathrm{ccm}$. Die erste Fällung wurde sofort abfiltriert.

\begin{tabular}{|c|c|c|c|c|c|c|}
\hline \multirow{2}{*}{$\begin{array}{c}\mathrm{CrCl}_{3} \cdot 6 \mathrm{H}_{2} \mathrm{O} \\
\mathrm{g}\end{array}$} & \multirow{2}{*}{$\begin{array}{l}\text { Essigsäure } \\
\text { in Mol, auf } \\
\text { je } 1 \text { Mol. } \\
\mathrm{CrCl}_{3} \cdot 6 \mathrm{H}_{2} \mathrm{O}\end{array}$} & \multicolumn{2}{|c|}{ Sofort gefällt } & \multicolumn{2}{|c|}{$\begin{array}{c}\text { Im Filtrat } \\
\text { abgeschieden }\end{array}$} & \multirow{2}{*}{$\begin{array}{c}\text { Gesamt } \\
\text { chlor } \\
\text { in } \%\end{array}$} \\
\hline & & $\mathrm{AgCl} \mathrm{g}$ & $\mathrm{Cl} \%$ & $\mathrm{AgCl} g$ & $\mathrm{Cl} \%$ & \\
\hline 0.2082 & 0 & 0.3350 & 39.79 & 0 & 0 & 39.79 \\
\hline 0.2108 & 1 & 0.3422 & 40.14 & 0 & 0 & 40.14 \\
\hline 0.2088 & 3 & 0.3370 & 39.91 & 0 & 0 & 39.91 \\
\hline 0.2052 & 5 & 0.3228 & 38.89 & 0.0106 & 1.27 & 40.16 \\
\hline 0.2194 & 7.1 & 0.3394 & 38.25 & 0.0156 & 1.76 & 40.01 \\
\hline 0.2100 & 33.2 & 0.2564 & 30.19 & 0.0810 & 9.54 & 39.73 \\
\hline 0.2092 & 111 & 0.2390 & 28.25 & 0.0992 & 11.72 & 39.97 \\
\hline 0.2076 & 449 & 0.2258 & 26.89 & 0.1090 & 12.98 & 39.87 \\
\hline 0.2088 & 670 & $0.269 \overline{8}$ & 31.95 & 0.0662 & 7.84 & 39.79 \\
\hline 0.2098 & 1111 & 0.2888 & 34.04 & 0.0484 & 5.70 & 39.74 \\
\hline
\end{tabular}

Die Fähigkeit der Essigsäure, die Menge des gefällten Chlors zu vermindern, ist, wie aus der vorstehenden Tabelle zu entnehmen, noch wesentlich kleiner als die der Milchsäure. Diese Wirkung der Sänren hängt daher mit ihrer Stärke zusammen. Auch durch sehr vermehrten Zusatz von Essigsäure kommt man nicht unter $2 / 3$ des Chlors herunter und dies wird erst erreicht bei etwa 400-500 Mol. (bei der Milchsäure durch $9 \mathrm{Mol}$.). Steigert man die Essigsäure noch mehr, so wird wieder mehr gefällt.

Eine bei gewöhnlicher Temperatur ausgeführte Versuchsreihe ergab die in der folgenden Tabelle $15 \mathrm{~b}$ enthaltenen Resultate.

Das niedrigste Chlor wurde wie bei den Versuchen bei $0^{\circ}$ bei 400-500 Mol. Essigsäure gefundén, aber es waren etwa $30 \%$, während bei $0^{0}$ nur etwa $27 \%(=2 / 3)$ niedergeschlagen worden waren. Auch sehr grolse Steigerung der Essigsäure vermehrt die Menge des gefällten Chlors nur wenig.

Zusatz geringerer Mengen Essigsäure (bis zu $3 \mathrm{Mol}$.). ist ohne Einflufs. Es wird dabei wie obne einen solchen (Tabelle 8, S. 305) sogleich das gesamte Chlor gefällt, (s. hierüber unten S. 318).

Falst man die bei den untersuchten Säuren erhaltenen Resultate zusammen, so zeigt sich zunächst, dafs die starken Säuren 
Tabelle $15 \mathrm{~b}$.

Chlorfällungen im grünen Chromchloridhydrat mit für 3 Atome Chlor berechnetem Silberacetat unter Zusatz steigender Menge Essigsä ure bei Zimmertemperatur und $50 \mathrm{ccm}$ Gesamtvolumen. Die erste Fällung wurde sofort abfiltriert.

\begin{tabular}{|c|c|c|c|c|c|c|}
\hline \multirow{2}{*}{$\begin{array}{c}\mathrm{CrCl}_{8} \cdot 6 \mathrm{H}_{2} \mathrm{O} \\
\mathrm{g}\end{array}$} & \multirow{2}{*}{$\begin{array}{l}\text { Essigsäure } \\
\text { in Mol, auf } \\
\text { je } 1 \text { Mol. } \\
\mathrm{CrCl}_{3} .6 \mathrm{H}_{2} \mathrm{O}\end{array}$} & \multicolumn{2}{|c|}{ Sofort gefällt } & \multicolumn{2}{|c|}{$\begin{array}{c}\text { Im Filtrat } \\
\text { abgeschieden }\end{array}$} & \multirow{2}{*}{$\begin{array}{c}\text { Gesamt- } \\
\text { chlor } \\
\text { in } \%\end{array}$} \\
\hline & & $\mathrm{AgCl} \mathrm{g}$ & $\mathrm{Cl} \%$ & $\mathrm{AgCl} \mathrm{g}$ & $\mathrm{Cl} \%$ & \\
\hline 0.2142 & 0 & 0.3446 & 39.78 & 0 & 0 & 39.78 \\
\hline 0.2132 & 0.12 & 0.3450 & 40.01 & 0 & 0 & 40.01 \\
\hline 0.2106 & 0.27 & 0.3402 & 39.94 & 0 & 0 & 39.94 \\
\hline 0.2104 & 0.36 & 0.3406 & 40.03 & 0 & 0 & 40.03 \\
\hline 0.2056 & 0.76 & $0 . \therefore 312$ & 39.83 & 0 & 0 & 39.83 \\
\hline 0.2084 & 1.5 & 0.3370 & 39.98 & 0 & 0 & 39.98 \\
\hline 0.2006 & 2.3 & 0.3236 & 39.89 & 0 & 0 & 39.89 \\
\hline 0.2130 & 7.3 & 0.3350 & 38.89 & 00102 & 1.18 & 40.07 \\
\hline 0.2108 & 31.6 & 0.2946 & 34.56 & 0.0458 & 5.37 & 39.93 \\
\hline 0.1976 & 67.4 & 0.2602 & 32.56 & 0.0598 & 7.48 & 40.04 \\
\hline 0.2034 & 109 & 0.2522 & 30.66 & 0.0772 & 9.38 & 40.04 \\
\hline 0.2138 & 175 & 0.2680 & 30.99 & 0.0778 & 8.99 & 39.98 \\
\hline 0.2082 & 450 & 0.2528 & 30.02 & 0.0848 & 10.07 & 40.09 \\
\hline 0.2118 & 665 & 0.2748 & 32.08 & 00688 & 8.03 & 40.11 \\
\hline 0.2144 & 1092 & 02628 & 30.31 & 0.0840 & 9.68 & 39.99 \\
\hline
\end{tabular}

Salpetersäure, Chlorsäure, Überchlorsäure, Übermangansäure die Menge des niedergeschlagenen Chlors am weitesten herunterdrängen, nämlich bis auf $1 / 3$ des Gesamtchlors $(13.3 \%$ ); die etwas weniger starke Schwefelsäure und die Milchsäure reduzieren das Chlor nur bis auf etwa $15 \%$ und bei der schwachen Essigsäure geht die Menge des gefällten Chlors nicht unter $2 / 3(26.6 \%)$ herab. Hiernach erscheint die Wirkung der Säuren im wesentlichen als eine solche ibrer Wasserstoffionen; damit steht in Übereinstimmung, dafs Essigsäure, in geringer Menge zugesetzt, ohne Einflufs ist (Tabelle $15 \mathrm{~b}$ oben), da die Konzentration ihrer Wasserstoffionen durch die Gegenwart des Silberacetats vermindert wird. Wäre es aber eine reine Wirkung der Wasserstoffionen, dann müfsten die in der angewandten Konzentration (bis zum Minimum von $13.3 \%$ Chlor) beinahe völlig dissoziierten starken Säuren Salpetersäure, Chlorsäure, Überchlorsäure, in derselben Molekülzahl zugesetzt, die Fällung derselben Chlorsilbermenge zur Folge haben. Dies ist aber nur annähernd der Fall, wie folgende Zusammenstellung zeigt: 
Mol. Säure auf

1 Mol. $\mathrm{CrCl}_{3} \cdot 6 \mathrm{H}_{2} \mathrm{O}$

0

0.05

0.08

0.14

0.25

0.81

1.3

2.5

3.5

6.0

13.0
$\%$ Cl gefällt bei Gegenwart von:

$\begin{array}{ccc}\mathrm{NO}_{3} \mathrm{H} & \mathrm{ClO}_{3} \mathrm{H} & \mathrm{ClO}_{4} \mathrm{H} \\ 31.6 & 30.9 & 28.7 \\ 29.6 & 24.6 & 26.9 \\ 28.1 & 20.7 & 26.7 \\ 26.3 & 19.6 & 26.4 \\ 23.0 & 18.0 & 25.3 \\ 17.1 & 15.2 & 21.2 \\ 16.2 & 14.5 & 17.6 \\ 14.8 & 14.0 & 14.1 \\ 14.4 & 13.8 & 14.0 \\ 14.1 & 13.7 & 13.8 \\ 14.0 & 13.7 & 13.7\end{array}$

Hier kommt aber in Betracht, dafs die bestreffenden Silbersalze allein ohne Säuren verschiedene Mengen Chlor niederschlagen, nämlich Silbernitrat (3 Mol.) 31.6\%, Silberchlorat 30.9\%, Silberperchlorat $28.7 \%$, (Tabelle 1 a, 3 u. 4, S. 299 ff.); aber auch wenn man dies berücksichtigt, ist die Wirkung gleicher Mol. der drei Säuren eine verschiedene.

Es ist somit neben der Stärke der Säure von dem Anion derselben abhängig, wieviel Chlor man erhält. Im grünen Chromchloridhydrat liegt ein Chlorid ror, gegen welches die einzelnen Silbersalze, besonders bei Gegenwart ihrer Säuren, sich verschieden verhalten, während sonst die Metallchloride mit allen Silbersalzen in gleicher Weise reagieren, da es lediglich auf das Silberion ankommt. Das grüne Chromchloridhydrat ist unseres Wissens bis jetzt das erste Chlorid, von dem gefunden wurde, dals es ein Reagens nicht allein auf das Silberion, sondern bei quantitativer Untersuchung auch auf das Anion des betr. Silbersalzes ist.

Bekanntlich hat Recoora ${ }^{1}$ ein dem grünen Chromchloridhydrat isomeres grauviolettes Salz darstellt, in welchem nach den Beobachtungen von WERNER und GUBSER durch Silbernitrat sogleich das gesamte Chlor gefällt wird. Wie wir konstatiert haben, ist dies bei diesem Chlorid der Fall, ob man Salpetersäure zusetzt oder nicht 'siehe S. 328). Dal's das grüne Chlorid in verdünnter wässeriger Lösung in das Violette übergeht, hat RECOURA auf thermochemischem und kolorimetrischem Wege, Werner durch Verfolgung der Änderung der elektrischen Leitfähigkeit nachgewiesen. Dals aber andererseits die Gegenwart von Salzsäure die Umwandlung des 
grünen in das violette Chlorid verhindert, oder wohl richtiger, dafs, je nach ihrer Menge die Bildung einer mehr oder weniger kleinen Menge violetten Chlorids möglich ist, haben Godefroy ${ }^{1}$ und RecourA ${ }^{2}$ gefunden; so erhält man nach Recoura beim Einleiten von Chlorwasserstoff in die wässerige, erhitzte und wieder erkaltete, Lösung des grünen Chlorids eine Abscheidung des violetten, ${ }^{3}$ beim Einleiten von Chlorwasserstoff in die salzsaure, erwärmte und wieder erkaltete, Lösung des grünen Chlorids eine solche eben des grünen Chlorids. Damit steht die von uns beobachtete, oben geschilderte, Wirkung der Säuren im Einklang; diese verlangsamen je nach ihrer Menge mehr oder weniger den Übergang des grünen Chlorids in das violette, und daher rührt es, dafs Silbersalze bei Zusatz von Säuren weniger Chlor fällen als ohne diesen. Es seien hier einige Versuche angeführt, bei welchen die Lösungen des Chlorids teils sogleich bei der Bereitung, teils erst vor der Fällung mit Salpetersäme versetzt wurden; vor der Fälllung blieben die Lösungen längere Zeit stehen. Die Menge der Salpetersäure betrug etwa 6 Mol., die des Silbernitrats etwa 4 auf $1 \mathrm{Mol}$. Chromchloridhydrat (etwa $0.2 \mathrm{~g}$ $\mathrm{CrCl}_{3} .6 \mathrm{H}_{2} \mathrm{O}, 0.5 \mathrm{~g} \mathrm{AgNO}_{3}, 0.3 \mathrm{~g} \mathrm{NO}_{3} \mathrm{H}$ ).

Tabelle 16 a.

Chlorfällungen im grünen Chromchloridhydrat. Die Lösungen des Chlorids wurden mit der Salpetersäure versetat und vor der Fällung bestimmte Zeit stehen gelassen. Gesamtvolumen $30 \mathrm{ccm}$. Die erste Fällung wurde sofort abfiltriert.

\begin{tabular}{|c|c|c|c|c|c|c|c|}
\hline \multirow{2}{*}{$\begin{array}{l}\mathrm{CrCl}_{3} . \\
6 \mathrm{H}_{2} \mathrm{O} \\
\quad \mathrm{g}\end{array}$} & \multirow{2}{*}{$\begin{array}{l}\text { Tem- } \\
\text { perat. } \\
\text { 0 }\end{array}$} & \multirow{2}{*}{$\begin{array}{l}\text { Zeit d. Stehen } \\
\text { d. Lösg. vor } \\
\text { d. Fällung }\end{array}$} & \multicolumn{2}{|c|}{ Erste Fällung } & \multicolumn{2}{|c|}{$\begin{array}{c}\text { Im Filtrat } \\
\text { abgeschieden }\end{array}$} & \multirow{2}{*}{$\begin{array}{l}\text { Gesamt- } \\
\text { chlor } \\
\text { in } \%\end{array}$} \\
\hline & & & $\mathrm{AgCl} \mathrm{g}$ & $\mathrm{Cl} \%$ & $\mathrm{AgCl} \mathrm{g}$ & CI $\%$ & \\
\hline 0.2130 & 0 & 3 Stunden & 0.1236 & 14.35 & 0.2204 & 25.68 & 39.93 \\
\hline 0.2076 & 0 & & 0.1424 & 16.96 & 0.1932 & 23.01 & 39.97 \\
\hline 0.1990 & 17 & $48 \quad$, & 0.2324 & 28.88 & 0.0886 & 11.01 & 39.89 \\
\hline 0.1966 & 17 & 50 Tage & 0.2492 & 31.34 & 0.0686 & 8.62 & 39.96 \\
\hline
\end{tabular}

Wie man sieht, konserviert die Säure das grüne Chlorid in der Lösung 3 Stunden lang fast unverändert, es wird noch etwa $1 / 3$ des Chlors gefällt, erst nach 48 Stunden zeigt sich die Umwandlung in das violette Chlorid deutlich, es werden $17 \%$ Chlor niedergeschlagen.

1 Compt. rend. 100 (1885), 105.

2. c. S. 37.

so wird dieses Chlorid auch dargestellt. 


\section{Tabelle $16 \mathrm{~b}$.}

Chlorfällungen im grünen Chromchloridhydrat. Die Lösungen des Chlorids wurden bestimmte Zeit stehen gelassen und dann mit dem Silbernitrat unter Zusatz der Salpetersäure gefällt. Gesamtrolumen $30 \mathrm{ccm}$. Erste Fällung sofort abfiltriert.

\begin{tabular}{|c|c|c|c|c|c|c|c|}
\hline \multirow{2}{*}{$\begin{array}{c}\mathrm{CrCl}_{3} . \\
6 \mathrm{H}_{2} \mathrm{O} \\
\mathrm{g}\end{array}$} & \multirow{2}{*}{$\begin{array}{l}\text { Tem- } \\
\text { perat. } \\
\text {. }\end{array}$} & \multirow{2}{*}{$\begin{array}{l}\text { Zeitd. Stehen } \\
\text { d. Lösg. vor } \\
\text { d. Fällung }\end{array}$} & \multirow{2}{*}{\multicolumn{2}{|c|}{$\begin{array}{l}\text { Erste Fällung } \\
\mathrm{AgCl} g \mid \mathrm{Cl} \%\end{array}$}} & \multicolumn{2}{|c|}{$\begin{array}{c}\text { Im Filtrat } \\
\text { abgeschieden }\end{array}$} & \multirow{2}{*}{$\begin{array}{c}\text { Gesamt } \\
\text { chlor } \\
\text { in } \%\end{array}$} \\
\hline & & & & & AgCl g & Cl $\%$ & \\
\hline & 0 & 3 Stunden & & 17.40 & & & 39.91 \\
\hline 0.2100 & 0 & $48 \quad$, & 0.2198 & 25.88 & 0.1188 & 13.99 & 39.87 \\
\hline 0.1908 & 17 & $48 \quad "$ & 0.2994 & 38.80 & 0.0098 & 1.27 & 40.07 \\
\hline 0.2152 & 17 & 50 Tage & 0.3444 & 39.57 & 0.0022 & 0.25 & 39.82 \\
\hline
\end{tabular}

Fügt man dagegen die Säure erst vor der Fällung hinzu, so wird viel mehr gefällt, nach 3 Stunden $17.4 \%$, nach 48 Stunden rund $26^{\circ} \%$. Bei gewöhnlicher Temperatur ist die Umwandlung eine raschere, wie die beiden letzten Versuche der Tabellen zeigen.

WERNER and GUBSER erteilen den beiden isomeren Chloriden anf Grund der elektrischen Leitfähigkeit, auf Grund der durch Silbernitrat gefällten Chlormengen bei Zusatz von Salpetersäure, sowie der Art des Wasserverlustes des grauvioletten Salzes (dieses geht bei vorsichtiger Entwässerung unter Verlust von 2 Mol. Wasser in das grüne $\mathrm{CrCl}_{3} \cdot 4 \mathrm{H}_{2} \mathrm{O}$ über) die Formeln:

$$
\left(\mathrm{Cr}_{4 \mathrm{H}_{2} \mathrm{O}}^{\mathrm{Cl}_{2}}\right) \mathrm{Cl}+2 \mathrm{aq}
$$

grünes Chlorid,

$$
\left[\mathrm{Cr}\left(\mathrm{H}_{2} \mathrm{O}\right)_{6}\right] \mathrm{Cl}_{3}
$$

grauviolettes Chlorid.

Man würde erwarten, dafs alle Silbersalze auch ohne Zusatz von Säure im grünen Chlorid $1 / 3$ des Chlors (die dem Chlorion entsprechende Menge) fällen sollten, denn durch Ersatz des Chlorions durch ein anderes Anion $\left(\mathrm{NO}_{3}, \mathrm{ClO}_{3}, \mathrm{ClO}_{4}\right.$ etc.) ändert sich am komplexen Kation nichts. In Wirklichkeit fällt jedes Silbersalz eine andere Menge Chlor. Manche wie das Acetat, wenn es in berechneter Menge, das Sulfat und das Laktat, wenn sie in einigem Überschufs hinzugefügt werden, das gesamte Chlor, keines nur $1 / 3$, sondern mindestens $2 / 3$. Dabei fällen gesteigerte Mengen der meisten Silbersalze (nicht des Perchlorats) mehr Chlor. Der Gleichgewichtszustand ${ }^{1}$

1 Nach den Bestimmungen der elektrischen Leitfähigkeit von WeRNER u. Gobser geht die Umwandlung des grünen in das violette Chlorid in wässeriger Lösung bei $0^{\circ}$ langsam vor sich. 
zwischen grünem und grauviolettem Chlorid ist unter diesen Umständen sowohl vom Anion des Silbersalzes, als von des letzteren Menge abhängig. Fällt ein Silbersalz sogleich das gesamte Chlor, so ist das Filtrat, wie zu erwarten, grau violett.

Nur bei Zusatz von starken Säuren in bestimmter Menge findet man bei der Fällung mit Silbersalzen das grüne Chlorid so dissoziiert, dafs nicht mehr als ein Chloratom als Ion fungiert. Auch hier verursacht bei einigen (z. B. Salpetersäure, Schwefelsäure) Steigerung der Säure, dafs mehr Chlor gefällt wird.

Wie sehr die Gegenwart von Wasserstoffionen auf die Erhaltung des grünen Chlorides von Einfluls ist, bewiesen Versuche in alkoholischer Lösung. Lösungen des Chlorids in starkem Alkohol reagieren, wie oben (S. 299) erwähnt, neutral. Fügt man zu ihnen auch bei $0^{0}$ alkoholisches Silbernitrat, so wird schon bei $6 \mathrm{Mol}$. davon auf 1 Mol. Chromchloridhydrat das gesamte Chlor sogleich gefällt.

Tabelle 17.

Chlorfällungen in grünen Chromchloridhydrat in alkoholischer Lösung mit steigenden Mengen alkoholischer Silbernitratlösung. Temperatur $0^{\circ}$. Gesamtvolumen $50 \mathrm{ccm}$. Erste Fällung sofort filtriert.

\begin{tabular}{|c|c|c|c|c|c|c|}
\hline \multirow{2}{*}{$\begin{array}{c}\mathrm{CrCl}_{3} \cdot 6 \mathrm{H}_{2} \mathrm{O} \\
\mathrm{g}\end{array}$} & \multirow{2}{*}{$\begin{array}{l}\text { Silbernitrat } \\
\text { berechnet für } \\
\text { Atome Chlor }\end{array}$} & \multicolumn{2}{|c|}{ Sofort gefällt } & \multicolumn{2}{|c|}{$\begin{array}{c}\text { Im Filtrat } \\
\text { abgeschieden }\end{array}$} & \multirow{2}{*}{$\begin{array}{l}\text { Gesamt- } \\
\text { chlor } \\
\text { in } \%\end{array}$} \\
\hline & & $\mathrm{AgCl} \mathrm{g}$ & $\mathrm{Cl} \%$ & $\mathrm{AgCl} \mathbf{g}$ & $\mathrm{Cl} \%$ & \\
\hline 0.15 & 3 & 0.2400 & 37.99 & 0.0120 & 1.90 & 39.89 \\
\hline 0.1098 & 6 & 0.1780 & 40.08 & 0 & 0 & 40.08 \\
\hline
\end{tabular}

Zusatz von Salpetersäure drückt auch unter diesen Umständen das Chlor herab, aber viel weniger weit als in wässeriger Lösung: 6-12 Mol. Salpetersäure reduzieren die Menge des gefällten Chlors nur bis auf $3 / 4$ des Gesamtchlors, mehr Säure fällt wieder mehr Chlor.

Tabelle 18.

Chlorfällungen im grünen Chromchloridhydrat, gelöst in absolutem Alkohol, mit für 3 Atome Chlor berechnetem Silbernitrat, ebenfalls in Alkohol gelöst, unter Zusatz steigender Mengen Salpetersä ure." Temperatur $0^{\circ}$. Gesamtvolumen $50 \mathrm{ccm}$. Erste Fällung sofort abfiltriert.

\begin{tabular}{|c|c|c|c|c|c|c|}
\hline \multirow{2}{*}{$\begin{array}{l}\mathrm{CrCl}_{3} \\
6 \mathrm{H}_{3} \mathrm{O} \\
\mathrm{g}\end{array}$} & \multirow{2}{*}{$\begin{array}{l}\text { Salpetersäure in } \\
\text { Mol. auf } 1 \mathrm{Mol} \text {. } \\
\mathrm{CrCl}_{\mathrm{B}} \cdot 6 \mathrm{H}_{2} \mathrm{O}\end{array}$} & \multicolumn{2}{|c|}{ Sofort gefällt } & \multicolumn{2}{|c|}{$\begin{array}{c}\text { Im Filtrat } \\
\text { abgeschieden }\end{array}$} & \multirow{2}{*}{$\begin{array}{l}\text { Gesamt- } \\
\text { chlor } \\
\text { in } \%\end{array}$} \\
\hline & & $\mathrm{AgCl} \mathrm{g}$ & Cl $\%$ & $\mathrm{AgCl} g$ & $\mathrm{Cl} \%$ & \\
\hline & $\mathbf{3}$ & 0.1284 & 33.28 & 0.0252 & 6.53 & 39.81 \\
\hline 0.1594 & 6 & 0.1930 & 29.94 & 0.0650 & 10.08 & 40.02 \\
\hline 0.1104 & 12 & 0.1368 & 30.64 & 0.0416 & 9.31 & 39.95 \\
\hline 0.1162 & 24 & 0.1640 & 34.89 & 0.0242 & 5.14 & 40.03 \\
\hline
\end{tabular}

$11 \mathrm{ccm}=0.33 \mathrm{~g} \mathrm{HNO}$. 


\section{Grünes Cäsiumchloriddoppelsalz, $\mathrm{CrCl}_{3} \cdot 2 \mathrm{CsCl}_{4} \mathrm{H}_{2} \mathrm{O}$.}

Dieses Salz wurde zuerst von Weuts und BouTwood ${ }^{1}$ dargestellt. $0.1514 \mathrm{~g}$ Substanz: $0.1918 \mathrm{~g} \mathrm{AgCl} .-0.2468 \mathrm{~g}$ Substanz: $0.0340 \mathrm{~g} \mathrm{Cr}_{2} \mathrm{O}_{3}$.

Berechnet für $\mathrm{CrCl}_{3} .2 \mathrm{CsCl}_{8} .4 \mathrm{H}_{2} \mathrm{O}$ :

$$
\begin{aligned}
& \mathrm{Cr}=9.19 \% \\
& \mathrm{Cl}=31.25 \%
\end{aligned}
$$

Gefunden:

9.43

31.32

Werner und Gobser erhielten durch Silbernitrat bei dem von ihnen beim Chromchloridhydrat angewandten Titrationsverfahren unter Zusatz von Salpetersäure (s. o. S. 307) $3 / 5$ des Chlors $(18.75 \%$ ) gefällt. Wir fanden, dafs auch hier ohne Zusatz von Salpetersäure mehr Chlor gefällt wird, und zwar durch die für 5 Atome Chlor berechnete Menge Silbernitrat $5 / 8$ des Chlors $(26.4 \%$ ). Beim Chromchloridhydrat wurden $\%$ des Chlors durch die für 3-4 Atome Chlor berechnete Menge Silbernitrat erhalten (s. Tabelle 1 a S.299).

Fügt man Salpetersäure hinzu, so sinkt die Menge des gefällten Chlors und man erhält bei Zusatz von $6 \mathrm{Mol}$. Säure auf $1 \mathrm{Mol}$. Doppelsalz $3 / 5$ des Gesamtchlors, d. h. die den beiden Chloratomen rom Cäsiumchlorid und dem einen Chloratom vom Chromchloridhydrat entsprechende Menge. Sie wird auch noch bei Gegenwart von 100 Mol. Salpetersäure niedergeschlagen. Beim Chromchloridhydrat allein wurde auch durch $6 \mathrm{Mol}$. Salpetersäure 1/3 des Chlors gefällt (8. Tabelle 9 a S. 306).

Tabelle 19.

Chlorfällungen im grünen $\mathrm{Chromch}$ lorid-Cäsiumchlorid $\mathrm{CrCl}_{8} .2 \mathrm{CsCl}_{4} 4 \mathrm{H}_{2} \mathrm{O}$ mit für 5 Atome Chlor berechnetem Silbernitrat unter Zusatz steigender Mengen Salpetersäure. Temperatur $0^{\circ}$. Gesamatvolumen $50 \mathrm{ccm}$. Erste Fällung sofort abfiltriert.

\begin{tabular}{c|c|c|c|c|c|c}
\hline \hline $\begin{array}{c}\mathrm{CrCl}_{\mathrm{g}} .2 \mathrm{CsCl} \\
4 \mathrm{H}_{2} \mathrm{O}\end{array}$ & $\begin{array}{c}\text { Salpetersäure } \\
\text { in Mol. auf } \\
\text { 1 Mol. } \\
\mathrm{g}\end{array}$ & \multicolumn{2}{|c|}{ Sofort gefällt } & \multicolumn{2}{|c|}{$\begin{array}{c}\text { Im Filtrat } \\
\text { abgeschieden }\end{array}$} & $\begin{array}{c}\text { Gesamt- } \\
\text { chlor } \\
\text { in \% } \%\end{array}$ \\
\hline \hline & $\mathrm{AgCl} \mathrm{g}$ & $\mathrm{Cl} \%$ & $\mathrm{AgCl} \mathrm{g}$ & $\mathrm{Cl} \%$ & \\
0.1104 & 0 & 0.1180 & 26.43 & 0.0224 & 5.01 & $31.44^{2}$ \\
0.1074 & 3 & 0.0886 & 20.40 & 0.0474 & 10.91 & 31.31 \\
0.0984 & 6 & 0.0784 & 19.70 & 0.0466 & 11.71 & 31.41 \\
0.1016 & 12 & 0.0780 & 18.98 & 0.0516 & 12.56 & 31.54 \\
0.1090 & 50 & 0.0832 & 18.87 & 0.0550 & 12.48 & 31.35 \\
0.1068 & 100 & 0.0820 & 18.98 & 0.0544 & 12.60 & 31.58 \\
\hline
\end{tabular}

2 Z. anorg. Chem. 10 (1895), 181. S. aucb Werner u. Gớrser l. c. S. 1602.

2 Berechnet für $3 \mathrm{Cl}: 18.75 \%$ für $5 \mathrm{Cl}: 31.25 \%$. 
Werner und Gubser legen dem Doppelsalz folgende Formel bei:

$$
\left(\begin{array}{c}
\mathrm{ClCs} \\
\mathrm{CrClCs} \\
\left(\mathrm{H}_{2} \mathrm{O}\right)_{4}
\end{array}\right) \mathrm{Cl}_{3} \text {. }
$$

$\mathrm{Da}$ in der wässerigen Lösung des Doppelsalzes das Chromchlorid sich gegen Silbernitrat mit und ohne Zusatz von Salpetersäure so verhält, wie wenn es allein darin vorhanden wäre (entsprechende Mengen Silbernitrat und Salpetersäure fällen dieselben Mengen Chlor im Doppelsalz wie im Chromchlorid), so ist es wahrscheinlich, dals das Doppelsalz in wässeriger Lösung in seine Komponenten Chromchlorid und Cäsiumchlorid zerfallen ist. Dies wird auch dadurch bestätigt, dafs man aus einer Lösung des Chromchlorids, welcher die für das Doppelsalz berechnete Menge Cäsiumchlorid hinzugefügt worden ist, durch Silbernitrat unter Zusatz von Salpetersäure dieselbe Menge Chlor gefällt erhält, wie aus der Lösung des Doppelsalzes, nämlich $3 / 5$ des Gesamtchlors.

\section{Tabelle 20.}

Chlorfällungen in Lösungen, welche grünes Chromchloridhydrat und Cüsiumehlorid in für das grüne Doppelsalu $\mathrm{CrCl}_{3} .2 \mathrm{CsCl}_{4} \mathrm{H}_{2} \mathrm{O}$ be rechneter Menge enthielten durch für 5 Atome Chlor berechnetes Silbernitrat unter Zusatz von Salpetersäure. Temperatur $0^{\circ}$. Gesamtrolumen $50 \mathrm{ccm}$.

Erste Fällung sofort abfiltriert.

\begin{tabular}{|c|c|c|c|c|c|c|}
\hline \multirow{2}{*}{$\begin{array}{l}\mathrm{CrCl}_{3} \cdot 6 \mathrm{H}_{2} \mathrm{O} \text { u. } \mathrm{CsCl} \text { in } \\
\text { dem Doppelsalz ent- } \\
\text { sprechenden Mengen }\end{array}$} & \multirow{2}{*}{$\begin{array}{l}\text { Sal- } \\
\text { peter- } \\
\text { saure } \\
\text { in Mol. }\end{array}$} & \multicolumn{2}{|c|}{ Sofort gefällt } & \multicolumn{2}{|c|}{$\begin{array}{c}\text { Im Filtrat } \\
\text { abgeschieden }\end{array}$} & \multirow{2}{*}{$\begin{array}{l}\text { Gesamt- } \\
\text { chlor } \\
\text { in } \%\end{array}$} \\
\hline & & $\mathrm{AgCl} \mathrm{g}$ & $\mathrm{Cl} \%$ & $\mathrm{AgCl} g$ & $\mathrm{Cl} \%$ & \\
\hline 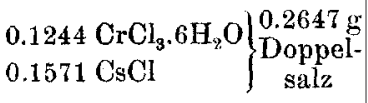 & 6 & 0.2102 & 19.64 & 0.1298 & 12.12 & 31.76 \\
\hline $\left.\begin{array}{c}0.1066 \mathrm{CrCl}_{3} \cdot 6 \mathrm{H}_{2} \mathrm{O} \\
0.1350 \mathrm{CsCl}\end{array}\right\} \begin{array}{c}0.2268 \mathrm{~g} \\
\text { Doppel- } \\
\text { salz }\end{array}$ & 12 & 0.1788 & 19.50 & 0.1128 & 12.30 & 31.80 \\
\hline
\end{tabular}

Überhaupt ändern anwesende Neutralsalze die Menge des im grünen Chromchloridhydrat fällbaren Chlors nicht; wir haben in dieser Richtung einen Versuch mit Kaliumnitrat gemacht und erhielten bei dessen Zusatz durch Fällung mit $3.9 \mathrm{Mol}$. $\mathrm{AgNO}_{3}$ auf $1 \mathrm{Mol}$. $\mathrm{CrCl}_{3} .6 \mathrm{H}_{2} \mathrm{O} \quad 34.5 \% \mathrm{Cl}$; aus der Tabelle 1 a S. $2 \bullet 9$ ist er- 
sichtlich, dafs durch 4 Mol. $\mathrm{AgNO}_{3} 33.9 \% \mathrm{Cl}$ niedergeschlagen werden.

$0.2036 \mathrm{~g}$ Chlorid lieferten bei Zusatz von $3.3 \mathrm{~g} \mathrm{KNO}_{3}$

aus dem Filtrat wurden erhalten

$$
\begin{aligned}
& 0.2842 \mathrm{~g} \mathrm{AgCl}=34.5 \% \mathrm{Cl} \\
& 0.0444 \mathrm{~g} \mathrm{AgCl}=\frac{5.4 \% \mathrm{Cl}}{39.9 \% \mathrm{Cl}}
\end{aligned}
$$

\section{Grünes Chrombromidhydrat, $\mathrm{CrBr}_{3} \cdot 6 \mathrm{H}_{2} \mathrm{O}$.}

Wir stellten dieses Bromid nach dem Verfahren von RECoURA ${ }^{1}$ aus Chromsäure her.

I. $0.3854 \mathrm{~g}$ Substanz: $0.0742 \mathrm{~g} \mathrm{Cr}_{2} \mathrm{O}_{3}--0.2872 \mathrm{~g}$ Substanz: $0.4042 \mathrm{~g} \mathrm{AgBr}$.

II. $0.3802 \mathrm{~g}$ Substanz: $0.0734 \mathrm{~g} \mathrm{Cr}_{2} \mathrm{O}_{\mathrm{s}}$. $-0.2282 \mathrm{~g}$ Substanz: $0.3218 \mathrm{~g} \mathrm{AgBr}$.

\begin{tabular}{ccc} 
Berechnet für & \multicolumn{2}{c}{ Gefunden: } \\
$\mathrm{CrBr}_{3}, 6 \mathrm{H}_{2} \mathrm{O}:$ & I & II \\
$\mathrm{Cr}=13.04$ & 13.18 & $13.22 \%$ \\
$\mathrm{Br}=59.95$ & 59.89 & 60.01
\end{tabular}

Es besteht wie das Chlorid aus sehr gut ausgebildeten, ungleich 6 seitigen Täfelchen von denselben optischen Eigenschaften wie jenes (S. 299). Es löst sich auch in Wasser mit saurer Reaktion, in starkem Alkohol neutral.

Werser und GOBser erhielten bei diesem Salz unter denselben Bedingungen wie beim Chlorid (Titration unter Zusatz von Salpetersäure, S. 307) $56.1 \%$ Brom $={ }^{14} / 15$ gefällt, Gesamtbrom berechnet $59.95 \%$.

Sämtliche von uns untersuchten Silbersalze fällen ohne Zusatz von Säure in berechneter Menge auch bei $0^{0}$ sogleich das gesamte Brom. Das Chlorid verhielt sich, wie oben (S. 299 ff.) beschrieben, ganz anders. In ihm fällen die Silbersalze starker Säuren auch in grofsem Überschufs nie das gesamte Chlor, nur Silberacetat tut dies.

Fügt man Salpetersäure hinzu, so sinkt die Menge des gefällten Broms und man erreicht ähnlich wie beim Chlorid bei etwa 6-9 Mol. Säure ein Minimum, welches aber weit über dem beim Chlorid beobachteten $1 / 3$ liegt. Man erhält als Minimum etwa $47 \%$ Brum gleich etwa $3 / 4$ des Gesamtbroms; steigert man die

1 Compt. rend. 110 (1890), 1029. S. auch Werner u. GUdBer, Ann. Chem. 322 (1902), 340.

Z. anorg. Chem. Bd. 39. 


\section{Tabelle 21.}

Bromfällungen im grünen Chrombromidhydrat durch verschiedene Silbersalze in für 3 Atome Brom berechneter Menge. Temperatur: $0^{\circ}$. Gesaintvolumen $50 \mathrm{ccm}$. Erste Fällung sofort abfiltriert.

\begin{tabular}{|c|c|c|c|c|c|c|}
\hline \multirow{2}{*}{$\begin{array}{l}\mathrm{CrBr}_{3} . \\
6 \mathrm{H}_{2} \mathrm{O} \\
\mathrm{g} \\
\end{array}$} & \multirow{2}{*}{$\begin{array}{c}\text { Silbersalz } \\
\text { für } 3 \text { Atome } \\
\text { Brom berechnet }\end{array}$} & \multicolumn{2}{|c|}{ Sofort gefällt } & \multicolumn{2}{|c|}{$\begin{array}{c}\text { Im Filtrat } \\
\text { abgeschieden }\end{array}$} & \multirow{2}{*}{$\begin{array}{l}\text { Gesamt- } \\
\text { brom } \\
\text { in } \%\end{array}$} \\
\hline & & $\mathrm{AgBr} g$ & $\mathrm{Br} \%$ & AgCl $\mathrm{g}$ & $\mathrm{Br} \%$ & \\
\hline 0.2090 & Silbernitrat & 0.2930 & 59.66 & 0 & 0 & $59.66^{1}$ \\
\hline 0.0683 & Silbersulfat & 0.0966 & 60.19 & 0 & 0 & 60.19 \\
\hline 0.1320 & Silberacetat & 0.1852 & 59.71 & 0 & 0 & 59.71 \\
\hline 0.1216 & Silberlaktat & 0.1708 & 59.77 & $\mathbf{0}$ & 0 & 59.77 \\
\hline 0.1251 & Silberchlorat & 0.1756 & 59.73 & 0 & 0 & $59.73^{2}$ \\
\hline 0.1234 & Silberperchlorat & 0.1734 & 59.80 & 0 & 0 & $59.80^{2}$ \\
\hline
\end{tabular}

Salpetersäure, so wächst auch die Menge des gefällten Broms wieder ziemlich stark und bei $72 \mathrm{Mol}$. werden $11 / 12$ daron niedergeschlagen. Beim Chlorid wurde bei Zusatz derselben Menge Salpetersäure nur wenig mehr als $1 / 3$ gefält (Tabelle 9 a S. 306).

\section{Tabelle 22.}

Bromfällungen im grünen Chrombromidhydrat mit für 3 Atome Brom berechnetem Silbernitrat unter Zusatz steigender Mengen Salpetersäure.

Temperatur $0^{\circ}$. Gesamtvolumen $50 \mathrm{ccm}$. Erste Fällung sofort filtriert.

\begin{tabular}{|c|c|c|c|c|c|c|}
\hline \multirow{2}{*}{$\begin{array}{c}\mathrm{CrBr}_{3} \cdot 6 \mathrm{H}_{2} \mathrm{O} \\
\mathrm{g}\end{array}$} & \multirow{2}{*}{$\mid \begin{array}{c}\mathrm{NO}_{8} \mathrm{H} \text { in Mol. } \\
\text { auf } 1 \mathrm{Mol} . \\
\mathrm{CrBr}_{3} \cdot 6 \mathrm{H}_{2} \mathrm{O}\end{array}$} & \multicolumn{2}{|c|}{ Sofort gefällt } & \multicolumn{2}{|c|}{$\begin{array}{c}\text { Im Filtrat } \\
\text { abgeschieden }\end{array}$} & \multirow{2}{*}{$\begin{array}{c}\text { Gesamt- } \\
\text { brom } \\
\text { in } \%\end{array}$} \\
\hline & & $\mathrm{AgBr} g$ & $\mathrm{Br} \%$ & $\mathrm{AgBr} \mathrm{g}$ & $\mathrm{Br} \%$ & \\
\hline 0.1524 & 0 & 0.2139 & 59.73 & 0 & 0 & 59.73 \\
\hline 0.2330 & 1 & 0.2934 & 53.59 & 0.0342 & 6.24 & 59.83 \\
\hline 0.1914 & 2 & 0.2190 & 48.69 & 0.0498 & 11.07 & 59.76 \\
\hline 0.2184 & 3 & 0.2512 & 48.95 & 0.0564 & 10.99 & 59.94 \\
\hline 0.2248 & 4 & 0.2512 & 47.55 & 0,0656 & 12.42 & 59.97 \\
\hline 0.1800 & 6 & 0.1990 & 47.05 & 0.0556 & 13.14 & 60.19 \\
\hline 0.1344 & 9 & 0.1474 & 46.67 & 0.0416 & 13.17 & 59.84 \\
\hline 0.2358 & 12 & 0.2772 & 50.03 & 0.0534 & 9.63 & 59.66 \\
\hline 0.2596 & 24 & 0.3218 & 52.75 & 0.0442 & 7.24 & 59.99 \\
\hline 0.1832 & 48 & 0.2276 & 52.87 & 0,0310 & 7.20 & 60.07 \\
\hline 0.2620 & 72 & 0.3378 & 54.87 & 0.0308 & 5.00 & 59.87 \\
\hline
\end{tabular}

1 Berechnet für $1 \mathrm{Br} 19.98 \%$, für $2 \mathrm{Br} 39.97 \%$, für $3 \mathrm{Br} 59.95 \%$.

2 Bei diesen Versuchen war das gebildete $\mathrm{AgBr}$ so schleimig, dafs es nicht filtriert werden konnte. Erst nach Zusatz von $2.0 \mathrm{~g}$ Natriumchlorat resp. Natriumperchlorat war dies möglich. 
Es wurden dann noch Fällungen mit Silberchlorat unter Zusatz von Chlorsäure und solche mit Silberperchlorat unter Zusatz von Überchlorsäure gemacht. Diese drücken das Brom weiter herunter als die Salpetersäure, die Chlorsäure beinahe bis zu $2 / 3$, die Überchlorsäure bis zu $\%$ (berechnet; $39.97 \% \mathrm{Br}$ ). Bei der Überchlorsäure ist es eigentümlich, dals die ersten $24 \mathrm{Mol}$. die Menge des gefällten Bromsilbers gar nicht vermindern, während $48 \mathrm{Mol}$. sie sogleich auf $2 / 3$ reduzieren. Beim Chlorid verhielten sich Chlorsäure und Überchlorsäure wie Salpetersäure, bei allen wurde von einer bestimmten Menge an $1 / 3$ des Chlors gefällt.

Tabelle 23.

Bromfällungen im grünen Chrombromidhydrat mit für 3 Atome Brom berechnetem Silberchlorat unter Zusatz steigender Mengen Chlorsäure. Temperatur $0^{\circ}$. Gesamtvolumen $50 \mathrm{ccm}$. Erste Fällung sofort filtriert.

\begin{tabular}{c|c|c|c|c|c|c}
\hline $\begin{array}{c}\mathrm{CrBr}_{8} .6 \mathrm{H}_{2} \mathrm{O} \mathrm{O} \\
\mathrm{g}\end{array}$ & $\begin{array}{c}\mathrm{HClO}_{\mathrm{g}} \text { in } \\
\text { Mol. a. 1 Mol. } \\
\text { Bromid }\end{array}$ & \multicolumn{2}{|c|}{ Sofort gefällt } & \multicolumn{2}{|c|}{$\begin{array}{c}\text { Im Filtrat } \\
\text { abgeschieden }\end{array}$} & $\begin{array}{c}\text { Gesamt- } \\
\text { brom } \\
\text { in } \%\end{array}$ \\
\hline \hline 0.1251 & 0 & 0.1756 & 59.73 & 0 & 0 & 59.73 \\
0.1138 & 3 & 0.1210 & 45.25 & 0.0386 & 14.44 & 59.69 \\
0.1228 & 6 & 0.1236 & 42.83 & 0.0488 & 16.91 & 59.74 \\
0.1042 & 12 & 0.1040 & 42.48 & 0.0432 & 17.64 & 60.12 \\
0.1224 & 24 & 0.1244 & 43.25 & 0.0480 & 16.69 & 59.94 \\
0.1298 & 48 & 0.1340 & 43.93 & 0.0496 & 16.26 & 60.19 \\
0.1298 & 96 & 0.1338 & 43.87 & 0.0498 & 16.33 & 60.20
\end{tabular}

Tabelle 24.

Bromfällungen im grünen Chrombromidhydrat mit für 3 Atome Brom berechnetem Silberperchlorat unter Zusatz ateigender Mengen Überchlorsäure. Temperatur $0^{\circ}$. Gesamtvolumen $50 \mathrm{ccm}$. Erste Fällung sofort filtriert.

\begin{tabular}{|c|c|c|c|c|c|c|}
\hline \multirow{2}{*}{$\begin{array}{c}\mathrm{CrBr}_{3} \cdot 6 \mathrm{H}_{2} \mathrm{O} \\
\mathrm{g}\end{array}$} & \multirow{2}{*}{$\begin{array}{c}\mathrm{HClO}_{4} \text { in } \mathrm{Mol} . \\
\text { auf } 1 \mathrm{Mol} . \\
\text { Bromid }\end{array}$} & \multicolumn{2}{|c|}{ Sofort gefällt } & \multicolumn{2}{|c|}{$\begin{array}{l}\text { Im Filtrat } \\
\text { abgeschieden }\end{array}$} & \multirow{2}{*}{$\begin{array}{c}\text { Gezamt } \\
\text { brom } \\
\text { in } \%\end{array}$} \\
\hline & & $\mathrm{AgBr} g$ & $\mathrm{Br} \%$ & $\mathrm{AgBr} \mathrm{g}$ & $\mathrm{Br} \%$ & \\
\hline 0.1516 & $3^{2}$ & 0.2134 & 59.94 & 0 & 0 & 59.94 \\
\hline 0.1026 & $6^{1}$ & 0.1444 & 59.89 & 0 & 0 & 59.89 \\
\hline 0.1150 & $12^{1}$ & 0.1615 & 59.76 & 0 & 0 & 59.76 \\
\hline 0.1242 & $24^{1}$ & 0.1742 & 59.68 & 0 & 0 & 59.68 \\
\hline 0.0964 & 48 & 0.0922 & 40.70 & 0.0438 & 19.34 & 60.03 \\
\hline 0.1086 & 96 & 0.1014 & 39.73 & 0.0514 & 20.14 & 59.87 \\
\hline
\end{tabular}

1 Bei diesen Fallungen war das abgeschiedene Bromsilber so schleimig, dals es erst nach Zusatz von je 2.0 Natriumperchlorat abfiltriert werden konnte. 
Die Werte der elektrischen Leitfähigkeit führen nach WERNER und Gubser wie beim Chlorid zu dem Schlufs, dafs das Bromid wie das Chlorid im ersten Moment der Lösung nur ein Bromion bilde:

$$
\left(\mathrm{Cr}_{4 \mathrm{H}_{2} \mathrm{O}}^{\mathrm{Br}_{2}}\right) \mathrm{Br}+2 \mathrm{aq} \text {. }
$$

Beim Chlorid waren die starken Säuren imstande, den Übergang des grünen in des grauviolette Salz bei Gegenwart von Silbersalzen so $\mathrm{zu}$ verlangsamen, dafs diese nur $1 / 3$ des Chlors fällten; beim Bromid vermögen sie dies nicht, hier werden mindestens $2 / 3$ niedergeschlagen.

Endlich seien noch einige Beobachtungen über diegrauvioletten Salze mitgeteilt.

Wir stellten das grauviolette Chlorid nach dem von WERNER und GUbser ${ }^{1}$ etwas abgeänderten Verfahren von RECourA dar, wir lösten wie jene Autoren das zum ersten Male abgeschiedene grauriolette Pulver wieder in wenig Wasser und leiteten in die unter $0^{\circ}$ abgekühlte Lösung Chlorwasserstoff, worauf sich das grauviolette Chlorid in schönen Kriställchen abschied.

Als wir mit demselben unter Zusatz von Salpetersäure genau wie beim grünen Chlorid Chlorfällungen machten, fanden wir, dals bei der ersten Fällung nicht alles Chlor niedergeschlagen wurde, etwa $1 \%$ war noch im Filtrat enthalten. Bestimmungen der elektrischen Leitfähigkeit, welche Herr DR. W. FenerLIN im hiesigen Institut auszuführen die Freundlichkeit hatte, lieferten etwas niedrigere Werte als sie WeRnER und Gubser erhielten. Wir vermuteten daher, dals das Salz noch etwas grünes Chlorid enthalte, und wiederholten die Reinigung durch Auflösen in wenig Wasser und Fällen durch Einleiten von Salzsäuregas noch 4 mal. In diesem Salz wurde durch etwas mehr als die berechnete Menge Silbernitrat mit und ohne Zusatz von Salpetersäure sogleich das gesamte Chlor bis auf Spuren gefällt; diese zeigten sich namentlich bei Zusatz von Salpetersäure im Filtrat als allmählich entstehende, nicht abfiltrierbare Opaleszenzen.

Was die Kristallform betrifft, so bildete das Salz unter dem Mikroskop meistens 4 oder 6 seitige, gut begrenzte Säulchen, bezw.

1 Ber. deutsch. chem. Ges. 34 (1901), 1591. 
Durchwachsungszwillinge oder -Drillinge, selten rautenförmige Täfelchen. Die Kristalle sind gerade auslöschend, das Achsenbild ist wegen ausserordentlicher Kleinheit und Hygroskopizität nicht zu beobachten (nach freundlicher Mitteilung von Herrn Dr. Pureninger).

I. $0.1664 \mathrm{~g}$ Substanz: $0.2672 \mathrm{~g} \mathrm{AgCl}$. $-0.3702 \mathrm{~g}$ Substanz: $0.1060 \mathrm{~g} \mathrm{Cr}_{\mathrm{z}} \mathrm{O}$.

II. $0.1700 \mathrm{~g}$ Substanz: $0.2734 \mathrm{~g} \mathrm{AgCl}$. $-0.2440 \mathrm{~g}$ Substanz: $0.0706 \mathrm{~g} \mathrm{Cr}_{2} \mathrm{O}_{3}$.

Berechnet für $\mathrm{CrCl}_{3} .6 \mathrm{H}_{2} \mathrm{O}$ :

$\mathrm{Cr}=19.54 \%$

$\mathrm{Cl}=39.90$,
Gefunden:

I II

$19.60 \quad 19.81$

$39.70 \quad 39.76$

Das grauviolette Bromid ist sogleich rein und liefert mit Silbernitrat auch bei Zusatz von Salpetersäure das gesamte Brom. Seine Kristallform stimmt mit der des Chlorids völlig überein.

$0.2504 \mathrm{~g}$ Substanz: $0.3526 \mathrm{~g} \mathrm{AgBr}$. - $0.2756 \mathrm{~g}$ Substanz: $0.0532 \mathrm{~g} \mathrm{Cr}_{2} \mathrm{O}_{3}$.

Berechnet für $\mathrm{CrBr}_{3} .6 \mathrm{H}_{2} \mathrm{O}$ :

$$
\begin{aligned}
& \mathrm{Cr}=13.04 \% \\
& \mathrm{Br}=59.95 \%
\end{aligned}
$$

Gefunden:

13.22

59.93

Die Lösung sowohl des grauvioletten Chlorids als des Bromids in Wasser reagiert sauer, die grauvioletten Salze sind wie die isomeren grünen hydrolytisch gespalten; die alkoholischen Lösungen der beiden Salze reagieren neutral, ebenso wie diejenigen der grünen.

' Werner und Gurser, Ann. Chem. 322 (1902), 343.

Tübingen, Ohemisches Institut, 25. März 1804.

Bei der Redaktion eingegangen am 27. März 1904. 